\title{
Decision Support System For Energy Source Selection Based On Credibility Fuzzy Information.
}

Saleem Abdullah ( $\square$ saleemabdullah@awkum.edu.pk)

Abdul Wali Khan University Mardan

Muhammad Yahya

Abdul Wali Khan University Mardan

\section{Research Article}

Keywords: Decision Support System, Energy Source, Credibility Fuzzy Information, Hamacher t-norm and t-conorm

Posted Date: December 16th, 2021

DOI: https://doi.org/10.21203/rs.3.rs-1054107/v1

License: (c) (1) This work is licensed under a Creative Commons Attribution 4.0 International License. Read Full License 


\title{
DECISION SUPPORT SYSTEM FOR ENERGY SOURCE SELECTION BASED ON CREDIBILITY FUZZY INFORMATION.
}

\author{
SALEEM ABDULLAH* AND MUHAMMAD YAHYA
}

\begin{abstract}
This main objective of this work is to define some new operations of credibility fuzzy numbers using Hamacher t-norm and t-conorm. These operation are more generalized operation for credibility fuzzy numnbers, we apply these operations to aggregation operators for credibility fuzzy numbers. Furthermore, using the basic operational laws of Hamacher t-norm and t-conorm, we develop a series of credibility fuzzy Hamacher aggregation operators like credibility fuzzy Hamacher weighted averaging (CFHWA) and credibility fuzzy Hamacher geometric (CFHWG) aggregation operators. we also explained some of the proposed Hamacher aggregation operators properties like commutativity, idempotency and monotonicity. In order to validate the proposed Hamacher aggregation operators for credibility fuzzy numbers, we develop general algorithm for decision making technique under credibility fuzzy numbers and using these operators. The proposed algorithm is apply to electricity crises in Pakistan problems. Finally a comparison with other existing methods is done to check the accuracy and validation of the proposed methods. At rest the proposed method is verified by other well known methods.
\end{abstract}

\section{INTRODUCTION}

Multi criteria decision making is method to decision making in which the best alternative is to find from the optimal decision in comparison to finite possibilities given a collection of many attributes. The MADM approach is gaining popularity among specialists since it can be used to a variety of fields, including operations research, engineering technology, management sciences and so on. Aggregation operators (AOs) play a critical role in solving MADM challenges by integrating data into a single useful form. Decision makers (DMs) employ various assessment techniques, Like crisp numbers or interval numbers, to rate the attributes in realworld decision-making. It got more difficult for the decision maker to using the real numbers (crisp number) as the data became more ambiguous and uncertain.

To address this issue, Zadeh [1] proposed fuzzy set (FS) having membership degree that membership degree belong to the close interval zero and one. With Zadeh's proposition of FS, DMs were able to tackle DMs issues involving less knowledge and errors with greater precision other than fuzzy set. Since, several aggregation operators in a fuzzy information were introduced to handle DMs issues. To defined and discussed various properties using fuzzy set the Song et al. [40] explained its idea in which main operational laws about fuzzy set were discussed. The induced generalized AOs and its application for solving the DMs issues using fuzzy set were introduced by Merigó and Gil-Lafuente [39].

Due to the ambiguity and difficulties of human conceptions for hard DM situations, current fuzzy DM approaches merely indicate fuzzy ways to support and lack degrees of credibility concerning the fuzzy provides strategic in the successful 
assessment of alternatives across variables. The majority of DM issues are made up of human realistic evaluations and ambiguous evaluations in uncertain and unclear settings. As a result, when DMs are more comfortable with certain measures but not with everyone, they might use varied degrees of credibility to generate more ambiguous evaluation values. In some research methodology frameworks, for example, each expert/reviewer is supposed to give each item an objective evaluation and related credibility score numbered from zero to 10 . For example, the expert can choose any value between 5 (equivalent to 0.5 fuzzy number) and 6 (equal to 0.6 fuzzy number), so it's obvious that value 0.6 is more credible (correct) than value 0.5. $(0.5,0.6)$. In this condition, they have fuzzy strategical assets as well, but they always decide their credibility degrees to maintain the credibility levels/degrees of the fuzzy evaluation values, because human decisions in uncertain and unspecified circumstances are never completely credible and correct. In an unclear context, the credibility measure of a fuzzy evaluation value must be closely tied to the fuzzy evaluation value, leading in more available and accurate assessment setting. As a result, this study develops the concept of a fuzzy credibility number (FCN), which is a new interpretation of the fuzzy concept in which a pair of fuzzy numbers represents both a fuzzy value and a credibility degree. So far, we've defined a series of proposed aggregation operators like CFHWA, CFHOWA, CFHHWA, CFHWG, CFHOWG and CFHHG

Originally developed by Dengs concept, the grey relational analysis (GRA) [37, 4] approach is used to a vast types of MADM issues. GRA's basic method entails converting the effectiveness of all alternatives into a series of comparisons. Grey system theory [37] is an approach for studying uncertainty that excels at mathematical modeling of systems with incomplete data. A system is called white, black respectively if the system is completely understood and unknown and incompletely unknown and understood respectively. Grey prediction, grey relational analysis (GRA), grey decision, grey programming and grey control are the five major aspects of the grey system theory. GRA is a type of grey system theory that can be used to resolve issues with complicated interactions among various components and variables. As a result, the GRA technique is commonly used to tackle ambiguity problems involving discrete data and unclear instructions $[5,6,7,8,9,10,11,12,13,14]$. Furthermore, the GRA technique is one of the most widely used approaches for analyzing diverse relationships among discrete data sets and making decisions in circumstances with multiple attributes. The GRA technique has several advantages, including the fact that the conclusions are based on original data, the calculation steps is easy, and it is one of the greatest approaches for making business decisions. To compare with exists methods like $[15,16,17,18,19,20,41,42]$ and $[23,24,25]$ GRA has been shown to be effective in handling problems with strong associations in MADM problems.

The TODIM (interactive and multicriteria decision making) approach was explained by Gomes and Lima [26, 27] [40, 41], and it was based on prospect theory [28]. The TODIM approach is used to determine the expected function, which calculates correct number information to determine the degree to which one alternative is preferable to another. Gomes and Rangel [29] were the first to effectively apply the TODIM method to the MADM, which is an easy and lowest step method for solving DMs issues. The TODIM approach was applied by Krohling and De Souza [30] to the criteria values, which are particularly stated as the IFS. Furthermore, 
the TODIM approach has proven to be quite useful in solving real-world situations. Fan et al. [47] improved the TODIM algorithm. TODIM algorithms will be able to deal with three forms of data in the future: real numbers, interval numbers, and triangular fuzzy numbers [32].

Fuzzy information aggregation operators are suitable and relevant in attractive research areas and are given strong consideration by researchers. There are different form of t-norm and t-conorm which is like Archimedean T-norms and T-conorms, Hamacher T-norms and T-conorms, Algebraic T-norms and T-conorms, Einstein T-norms and T-conorms, Frank T-norms and T-conorms and Dombi T-norms and T-conorms. Zhou and Chen [33] created a new distance measure for PFSs as well as several new similarity measures. Mohagheghi et al. [34] looked at several novel aggregation operators in PFSs and looked at how they could be used in MCGDM situations. The Hamacher T-conorm and T-norm, which are extensions of algebraic and Einstein T-conorm and T-norm, are more valid and flexible [35]. The value of study on aggregation operators using Hamacher operations and their applications to MADM issues is considerable. Augustine [36] max-min composition was established for PFSs and used in field of study placement based on academic ability. For multi-attribute decision-making, Tan et al. [37] developed hesitant fuzzy Hamacher aggregation operations. Senapati and Yager [38] Fermatean fuzzy weighted average (FFWA) operator, Fermatean fuzzy weighted geometric (FFWG) operator, Fermatean fuzzy weighted power average (FFWPA) operator, and Fermatean fuzzy weighted power geometric (FFWPG) operator are four new types of weighted aggregation operators for FFS.

For carrying out the combination process, all of the following operators are based on the algebraic operational laws of FCNs, which are incompatible with the limiting case of regular fuzzy sets. However, these operators are incapable of aggregating the fuzzy credibility data. As a result, figuring out how to extend the Hamacher operations to aggregate fuzzy credibility data is a worthwhile project, which is also the topic of this study. Due to the fact that there are many scholars working in the subject of fuzzy sets, he used all forms and generalizations in his research. The basic Hamacher t-norm and t-conorm can be used to generate fuzzy credibility numbers and their basic working principles. The following is a summary of one of the paper's key goals.

- As in the proposed work we have taken the data in the form FCNs which will provide us that numbers which is actually a fuzzy number and its degree of credibility(accurateness) which is also taken from the close interval zero to one. The other thing is that there is no further need that its total or combination be equal to one. There are no conditions for fuzzy numbers, so we can take them as we choose. We can also take the degree of credibility, which indicates how accurate the associated fuzzy number is in comparison to others.

- We may take the Hamacher t-norm and t-conorm, which we will describe as the core operational laws, and create a series of aggregation operators based on their Hamacher operational laws, which will provide a high and broad range for decision making issues. Another feature is that the Hamacher t-norm and t-conorm are used to organize data in a certain format while also assisting in the removal of ambiguity and vagueness in operational laws.

- The third and most significant purpose is to establish a solid connection between the proposed model and MCGDM difficulties. Under the influence of the 
suggested model's operator estimate approaches, we offer novel operators for determining MCGDM concerns. To illustrate the usefulness and effectiveness of the desired solution, a useful example applicable to select and study the electricity crises in Pakistan.

In section II of the article, we explained a score function and accuracy function for FCNs. And in section III we have explained the Hamacher operation, Hamacher operation for FCNs. We also suggested average and geometric aggregation operators including CFHWA, CFHOWA, CFHHWA, CFHWG, CFHOWG and CFHHG in sections IV, V VI, VII, VIII, IX and X. We have explained an extended GRA and TODIM approach frameworks for MCGDM, as well as their step-by-step algorithms and a numerical model using the extended GRA and TODIM methods for studying the electricity crises in Pakistan is presented in section XI. A comparison of the essential approach is to verify with such previous approaches is presented lastly, implying that the proposed model is also more successful than the existing works.

\section{Preliminaries}

The basic concepts about the FS and FCNs are presented in this section, which will be useful in certain studies.

Let $X \neq \phi$.Then, the FS $A$ in $X$ is define as [1],

$$
A=\left\{\left\langle x, u_{A}(x)\right\rangle \mid x \in X\right\}
$$

where $u_{A}(x)$ show the degree of membership and also $u_{A}(x)$ contain to the close interval zero and one.

Let $\check{N} \neq \phi$. Then, the FCNs $\tilde{I}$ is defined as [2],

$$
\tilde{I}=\{\langle x, \bar{u}(t), v(s)\rangle \mid x \in X\},
$$

where $\bar{u}(t) \rightarrow[0,1], v(s) \rightarrow[0,1]$ are the membership function of $x$ in $\tilde{I}$ and the degree of credibility related to $\bar{u}(t)$ respectively, Then the pair $\left(x, \bar{u}_{t}, v_{s}\right)$ are called fuzzy credibility numbers (FCNs). Consider $s_{1}=\left(u_{1}, c_{1}\right)$ and $s_{2}=\left(u_{2}, c_{2}\right)$ are FCNs then we have,

(1) $s_{1} \supseteq s_{2} \Leftrightarrow u_{1} \supseteq u_{2}, c_{1} \supseteq c_{2}$

(2) $s_{1}=s_{2} \Leftrightarrow s_{1} \supseteq s_{2}, s_{2} \supseteq s_{1}$

(3) $s_{1} \cup s_{2} \Leftrightarrow\left(u_{1} \vee u_{2}, c_{1} \vee c_{2}\right)$

(4) $s_{1} \cap s_{2} \Leftrightarrow\left(u_{1} \wedge u_{2}, c_{1} \wedge c_{2}\right)$

(5) $\left(s_{1}\right)^{c}=\left(1-u_{1}, 1-c_{1}\right)$

In [2] defined the operational laws but here we define another operational laws for fuzzy credibility numbers.

Consider three FCNs $\alpha_{j}, \alpha_{1}, \alpha_{2}$ Then we have,

(1) $\alpha_{1} \oplus^{*} \alpha_{2}=\left(u_{1}+u_{2}-u_{1} u_{2}, c_{1}+c_{2}-c_{1} c_{2}\right)$

(2) $\alpha_{1} \otimes^{*} \alpha_{2}=\left(u_{1} u_{2}, c_{1} c_{2}\right)$

(3) $\lambda^{\alpha^{*}}=\left(1-\left(1-u_{1}\right)^{\lambda}, c^{\lambda}\right)$, for $\lambda>0$.

(4) $\alpha^{* \lambda}=\left((u)^{\lambda}, 1-(1-c)^{\lambda}\right)$, for $\lambda>0$.

Consider $s_{i}=\left(u_{i}, c_{i}\right)$ is a FCNs then the score function is defined as follow,

$$
S\left(s_{i}\right)=\left[u_{i} c_{i}+\left(u_{i}+c_{i}\right) / 2\right] / 2
$$




\section{Hamacher Operators}

The terms t-norm and t-conorm are used in fuzzy set theory to construct a generalized union and intersection of fuzzy sets [41]. The definitions and explanation of t-norm and t-conorm were given by Roychowd Hury and Wang [42]. Deschrijver and Kerre [43] proposed a generalized union and a generalized intersection of IFSs based on a t-norm and t-conorm. Hamacher is a basically more generalized form of t-norm and t-conorm. Also using the Hamacher t-norm and t-conorm we developed various types of Hamacher operations which is presented in the below section of the paper. The Hamacher product $\otimes$ is a t-norm and Hamacher sum $\oplus$ is a t-conorm which can be written as,

$$
\begin{gathered}
T\left(a_{i}, b_{i}\right)=a \otimes b=\frac{a b}{\lambda+(1-\lambda)(a+b-a b)} \\
T *\left(a_{i}, b_{i}\right)=a \oplus b=\frac{a+b-a b-(1-\lambda) a b}{1-(1-\lambda) a b}
\end{gathered}
$$

\section{Hamacher Aggregation Operators For Credibility Fuzzy Numbers}

This section provide the new work on fuzzy credibility numbers. First we define the Hamacher operational laws for FCNs. These operational law will use to define some aggregation operator for FCNs. We developed fuzzy credibility Hamacher averaging and geometric aggregation operators.

4.1. Hamacher Operators For Credibility Fuzzy Set. In this subsection we can defined the Hamacher operational laws which are used further in this study. On the basis of these operational laws of Hamacher t-norm and t-conorm we can proposed a series of aggregation operators. And also by using these aggregation operators (Aops) we can select the good (best) option (alternatives).

In the previous work the basic properties of Hamacher aggregation operators using different form of fuzzy set. But in this paper we have define the new basic properties for FCNs using Hamacher operational laws which is as under.

(1) $\alpha_{1} \oplus^{*} \alpha_{2}=\left(\frac{a_{1}+a_{2}-a_{1} a_{2}-(1-\lambda) a_{1} a_{2}}{1-(1-\lambda) a_{1} a_{2}}, \frac{b_{1} b_{2}}{\lambda+(1-\lambda)\left(b_{1}+b_{2}-b_{1} b_{2}\right)}\right)$.

(2) $\alpha_{1} \otimes^{*} \alpha_{2}=\left(\frac{a_{1} a_{2}}{\lambda+(1-\lambda)\left(a_{1}+a_{2}-a_{1} a_{2}\right)}, \frac{b_{1}+b_{2}-b_{1} b_{2}-(1-\lambda) b_{1} b_{2}}{1-(1-\lambda) b_{1} b_{2}}\right)$.

(3) $\beta . \alpha_{1}^{*}=\left(\frac{\left(1+a_{i}\right)^{\beta}-\left(1-a_{i}\right)^{\beta}}{\left(1+a_{i}\right)^{\beta}+\left(1-a_{i}\right)^{\beta}}, \frac{2 \cdot b_{i}^{\beta}}{\left(2-b_{i}\right)^{\beta}+b_{i}^{\beta}}\right)(\beta>0)$.

(4) $\alpha_{1}^{*^{\beta}}=\left(\frac{2 . a_{i}^{\beta}}{\left(2-a_{i}\right)^{\beta}+a_{i}^{\beta}}, \frac{\left(1+b_{i}\right)^{\beta}-\left(1-b_{i}\right)^{\beta}}{\left(1+b_{i}\right)^{\beta}+\left(1-b_{i}\right)^{\beta}}\right)(\beta>0)$

\section{Credibility Fuzzy Hamacher Averaging Aggregation Operator}

This subsection provide us the detail discussion of new designed credibility fuzzy Hamacher averaging operator, By using the Hamacher operational law (1) and (3), We can developed credibility fuzzy averaging operator. Assume that $s_{i}(i=1, \ldots, n)$ is a set of FCNs in $\grave{U} \neq \phi$. Then the mapping $\alpha^{n} \rightarrow \alpha$, is said to be CFHWA operator.

$$
C F H W A\left(s_{1}, s_{2}, \ldots, s_{n}\right)=\oplus_{i=1}^{n} s_{i} w_{i}
$$

The weight vector is represented by $w=\left(w_{1}, w_{2}, \ldots, w_{n}\right)^{T}$, with $\Sigma_{i=1}^{n} w_{i}=1$ and $w_{i} \in[0,1]$. Using the CFHWA operator, the aggregated value of $s_{i}$ becomes an 
FCNs, which can be described as;

$$
\begin{aligned}
& C F H W A\left(s_{1}, s_{2}, \ldots, s_{n}\right) \\
= & \left\{\oplus_{i=1}^{n} s_{i} w_{i}\right\} \\
= & \left\{\begin{array}{c}
\frac{\Pi_{i=1}^{n}\left(1+(\lambda-1) a_{i}\right)^{w_{i}}-\Pi_{i=1}^{n}\left(1-a_{i}\right)^{w_{i}}}{\prod_{i=1}^{n}\left(1+(\lambda-1) a_{i}\right)^{w_{i}}+(\lambda-1) \Pi_{i=1}^{n}\left(1-a_{i}\right)^{w_{i}}} \\
\frac{\lambda \Pi_{i=1}^{n} b_{i}^{w_{i}}}{\Pi_{i=1}^{n}\left(1+(\lambda-1)\left(1-b_{i}\right)\right)^{w_{i}}+(\lambda-1) \Pi_{i=1}^{n} b_{i}^{w_{i}}}
\end{array}\right\}
\end{aligned}
$$

In the next theorem we prove that the equation 5.1 is also a FCNs and the aggregated value of FCNs $\vartheta=\left\{s_{i}, i=1,2, \ldots, n\right\}$ is also a FCNs.

Theorem 1. Consider a collection of $\vartheta=\left\{s_{i}, i=1,2, \ldots, n\right\}$ is a FCNs having weight vector $w=\left(w_{1}, w_{2}, \ldots, w_{n}\right)^{T}$. Then the aggregated value of $\vartheta=\left\{s_{i}, i=\right.$ $1,2, \ldots, n\}$ is also a FCNs.

$$
\begin{aligned}
& C F H W A\left(s_{1}, s_{2}, \ldots, s_{n}\right) \\
= & \left\{\oplus_{i=1}^{n} s_{i} w_{i}\right\} \\
= & \left\{\begin{array}{c}
\frac{\Pi_{i=1}^{n}\left(1+(\lambda-1) a_{i}\right)^{w_{i}}-\Pi_{i=1}^{n}\left(1-a_{i}\right)^{w_{i}}}{\Pi_{i=1}^{n}\left(1+(\lambda-1) a_{i}\right)^{w_{i}+(\lambda-1)}} \\
\frac{\lambda \Pi_{i=1}^{n} b_{i}^{w_{i}}}{\Pi_{i=1}^{n}\left(1-a_{i}\right)^{w_{i}}} \\
\frac{\Pi_{i=1}^{n}\left(1+(\lambda-1)\left(1-b_{i}\right)\right)^{w_{i}}+(\lambda-1) \Pi_{i=1}^{n} b_{i}^{w_{i}}}{n}
\end{array}\right\}
\end{aligned}
$$

Proof. Using mathematical induction we have to proof the above theorem,

$$
\begin{aligned}
& s_{1}+s_{2} \\
& =\left\{\begin{array}{c}
\frac{\Pi_{i=1}^{n}\left(1+(\lambda-1) a_{i}\right)^{w_{i}}-\Pi_{i=1}^{n}\left(1-a_{i}\right)^{w_{i}}}{\Pi_{i=1}^{n}\left(1+(\lambda-1) a_{i} w_{i}+(\lambda+1) \Pi_{i=1}^{n}\left(1-a_{i}\right)^{w_{i}}\right.}, \\
\frac{\lambda \Pi_{i=1}^{n} b_{i}^{w_{i}}}{\Pi_{i=1}^{n}\left(1+(\lambda-1)\left(1-b_{i}\right)\right)^{w_{i}}+(\lambda-1) \Pi_{i=1}^{n} b_{i}^{w_{i}}}
\end{array}\right\} \\
& =\left\{\begin{array}{c}
\frac{\left(1+(\lambda-1) a_{1}\right)^{w_{1}}\left(1+(\lambda-1) a_{2}\right)_{2}-\left(1-a_{1}\right)^{w_{1}}\left(1-a_{2}\right)^{w_{2}}}{\left(1+(\lambda-1) a_{1}\right)^{w_{1}}\left(1+(\lambda-1) a_{2}\right)^{w_{2}}\left(1+(\lambda-1)\left(1-a_{1}\right)^{w_{1}}\left(1-a_{2}\right)^{w_{2}}\right.}, \\
\frac{\lambda b_{i}^{w_{i}} b_{2}^{w_{i}}}{\left(1+(\lambda-1)\left(1-b_{1}\right)\right)^{w_{1}}\left(1+(\lambda-1)\left(1-b_{2}\right)\right)^{w_{2}}+(\lambda-1) b_{1}^{w_{1}} b_{2}^{w_{2}}}
\end{array}\right\} \\
& =\left\{\begin{array}{c}
\frac{\Pi_{i=1}^{2}\left(1+(\lambda-1) a_{i}\right)^{w_{i}}-\Pi_{i=1}^{2}\left(1-a_{i}\right)_{i}}{\Pi_{i=1}^{2}\left(1+(\lambda-1) a_{i}\right)_{i}^{w_{i}}+(\lambda-1) \Pi_{i=1}^{2}\left(1-a_{i}\right)^{w_{i}}}, \\
\frac{\lambda \Pi_{i=1}^{2} b_{i}^{w_{i}}}{\Pi_{i=1}^{2}\left(1+(\lambda-1)\left(1-b_{i}\right)\right)^{w_{i}}+(\lambda-1) \Pi_{i=1}^{2} b_{i}^{w_{i}}}
\end{array}\right\}
\end{aligned}
$$

Let $n=2$, then,

$$
\begin{aligned}
& C F H W A\left(s_{1}, s_{2}\right) \\
= & \left\{\oplus_{i=1}^{2} s_{i} w_{i}\right\} \\
= & \left\{\begin{array}{c}
\frac{\Pi_{i=1}^{2}\left(1+(\lambda-1) a_{i}\right)^{w_{i}}-\Pi_{i=1}^{2}\left(1-a_{i}\right)^{w_{i}}}{\Pi_{i=1}^{2}\left(1+(\lambda-1) a_{i}\right)^{w_{i}}+(\lambda-1) \Pi_{i=1}^{2}\left(1-a_{i}\right)^{w_{i}}} \\
\frac{\lambda \Pi_{i=1}^{2} b_{i}^{w_{i}}}{\Pi_{i=1}^{2}\left(1+(\lambda-1)\left(1-b_{i}\right)\right)^{w_{i}}+(\lambda-1) \Pi_{i=1}^{2} b_{i}^{w_{i}}}
\end{array}\right\}
\end{aligned}
$$

Which will prove that the for $n=2$ we have true the statement and now for $n=k$ we have to check.

$$
\begin{aligned}
& C F H W A\left(s_{1}, s_{2}, \ldots, s_{k}\right) \\
= & \left\{\begin{array}{c}
\frac{\Pi_{i=1}^{k}\left(1+(\lambda-1) a_{i}\right)_{i}-\Pi_{i=1}^{k}\left(1-a_{i}\right)^{w_{i}}}{\Pi_{i=1}^{k}\left(1+(\lambda-1) a_{i}\right)^{w_{i}}+\left(\lambda-1 \Pi_{i=1}^{k}\left(1-a_{i}\right)^{w_{i}}\right.}, \\
\frac{\lambda \Pi_{i=1}^{k} b_{i}^{w_{i}}}{\Pi_{i=1}^{k}\left(1+(\lambda-1)\left(1-b_{i}\right)\right)^{w_{i}}+(\lambda-1) \Pi_{i=1}^{k} b_{i}^{w_{i}}}
\end{array}\right\}
\end{aligned}
$$


Further, we check for $n=k+1$, we have,

$$
\begin{aligned}
& C F H W A\left(s_{1}, s_{2}, \ldots, s_{k+1}\right) \\
& =\left\{\begin{array}{c}
\frac{\Pi_{i=1}^{n}\left(1+(\lambda-1) a_{i}\right)^{w_{i}}-\Pi_{i=1}^{n}\left(1-a_{i}\right)^{w_{i}}}{\Pi_{i=1}^{n}\left(1+(\lambda-1) a_{i}\right)^{w_{i}+(\lambda-1) \Pi_{i=1}^{n}\left(1-a_{i}\right)^{w_{i}}}}, \\
\frac{\lambda \Pi_{i=1}^{n} b_{i}^{w_{i}}}{\Pi_{i=1}^{n}\left(1+(\lambda-1)\left(1-b_{i}\right)\right)^{w_{i}}+(\lambda-1) \Pi_{i=1}^{n} b_{i}^{w_{i}}}
\end{array}\right\} \\
& =\left\{\begin{array}{c}
\frac{\left(1+(\lambda-1) a_{k}\right)^{w_{k}}\left(1+(\lambda-1) a_{k+1}\right)^{w_{k+1}}-\left(1-a_{k}\right)^{w_{k}}\left(1-a_{k+1}\right)^{w_{k+1}}}{\left(1+(\lambda-1) a_{k}\right)^{w_{k}}\left(1+(\lambda-1) a_{k+1}\right)^{w_{k}+1}+(\lambda-1)\left(1-a_{k}\right)^{w_{k}}\left(1-a_{k+1}\right)^{w_{k+1}}}, \\
\frac{\lambda b_{k}^{w_{k}} b_{k+1}^{w_{k+1}}}{\left(1+(\lambda-1)\left(1-b_{k}\right)\right)^{w_{k}}\left(1+(\lambda-1)\left(1-b_{k+1}\right)\right)^{w_{k+1}}+(\lambda-1) b_{k}^{w_{k}} b_{k+1}^{w_{k+1}}}
\end{array}\right\} \\
& =\left\{\begin{array}{c}
\frac{\Pi_{i=1}^{k+1}\left(1+(\lambda-1) a_{i}\right)^{w_{i}}-\Pi_{i=1}^{k+1}\left(1-a_{i}\right)^{w_{i}}}{\Pi_{i=1}^{k+1}\left(1+(\lambda-1) a_{i}\right)^{w_{i}}+(\lambda-1) \Pi_{i=1}^{k+1}\left(1-a_{i}\right)^{w_{i}}} \\
\frac{\lambda \Pi_{i=1}^{k+1} b_{i}^{w_{i}}}{\Pi_{i=1}^{k+1}\left(1+(\lambda-1)\left(1-b_{i}\right)\right)^{w_{i}}+(\lambda-1) \Pi_{i=1}^{k+1} b_{i}^{w_{i}}}
\end{array}\right\}
\end{aligned}
$$

The result is true for $\mathrm{n}=\mathrm{k}+1$ and hence true for $n \geq 1$.

In the next theorem we discuss some characteristic of the CFHWA operator. Here also the basic properties like monotonicity, boundedness and idempotency using FCNs and Hamacher t-norm and t-norm.

Theorem 2. Consider a collection of $\vartheta=\left\{s_{i}, i=1,2, \ldots, n\right\}$ is a FCNs having weight vector $w=\left(w_{1}, w_{2}, \ldots, w_{n}\right)^{T}$. Then we have following properties.

(1) (The idempotency): If $s_{i}=s=(u, c) \forall i=1, \ldots, n$. Then,

$$
C F H W A\left(s_{1}, s_{2}, \ldots, s_{n}\right)=s
$$

(2) (Boundedness): If $s_{i}(i=1, \ldots, n)^{-}$is a group of FCNs then a set $s_{\min }=$ $\left(\min _{i} u_{i}, \max _{i} c_{i}\right)$ and $s_{\max }=\left(\max _{i} u_{i}, \min _{i} c_{i}\right)$ are the maximum and minimum FCNs. Then,

$$
s_{\min } \leq C F H W A\left(s_{1}, s_{2}, \ldots, s_{n}\right) \leq s_{\max }
$$

(3) (Monotonicity): Let $s_{i}(i=1, \ldots, n), s_{i}^{*}(i=1, \ldots, n)$ are the family of FCNs such that $s_{i} \leq s_{i}^{*}$. Thus,

$$
\begin{array}{r}
C F H W A\left(s_{1}, s_{2}, \ldots, s_{n}\right) \\
\leq \quad C F H W A\left(s_{1}^{*}, s_{2}^{*}, \ldots, s_{n}^{*}\right)
\end{array}
$$

Proof. (1). We have $s_{i}=s=(u, c)(i=1, \ldots, n)$, which give the results.

$$
\begin{aligned}
& C F H W A\left(s_{1}, s_{2}, \ldots, s_{n}\right) \\
= & \left\langle\oplus_{i=1}^{n} s_{i} w_{i}\right\rangle \\
= & \left\{\begin{array}{c}
\frac{\Pi_{i=1}^{n}\left(1+(\lambda-1) a_{i}\right)^{w_{i}}-\Pi_{i=1}^{n}\left(1-a_{i}\right)^{w_{i}}}{\Pi_{i=1}^{n}\left(1+(\lambda-1) a_{i}\right)^{w_{i}}+(\lambda-1) \Pi_{i=1}^{n}\left(1-a_{i}\right)^{w_{i}}} \\
\frac{\lambda \Pi_{i=1}^{n} b_{i}^{w_{i}}}{\Pi_{i=1}^{n}\left(1+(\lambda-1)\left(1-b_{i}\right)\right)^{w_{i}}+(\lambda-1) \Pi_{i=1}^{n} b_{i}^{w_{i}}}
\end{array}\right\}
\end{aligned}
$$


For all $i$. Therefore,

$$
\begin{aligned}
& =\left\{\begin{array}{c}
\frac{\Pi_{i=1}^{n}\left(1+(\lambda-1) a_{i}\right)^{w_{i}}-\Pi_{i=1}^{n}\left(1-a_{i}\right)^{w_{i}}}{\Pi_{i=1}^{n}\left(1+(\lambda-1) a_{i}\right)^{w_{i}}+(\lambda-1) \Pi_{i=1}^{n}\left(1-a_{i}\right)^{w_{i}}}, \\
\frac{\lambda \Pi_{i=1}^{n} b_{i}^{w_{i}}}{\Pi_{i=1}^{n}\left(1+(\lambda-1)\left(1-b_{i}\right)\right)^{w_{i}}+(\lambda-1) \Pi_{i=1}^{n} b_{i}^{w_{i}}}
\end{array}\right\} \\
& =\left\{\left(1-(1-u)^{\sum_{k=1}^{n} p_{k}}\right),\left(\left(1-(1-c)^{\sum_{k=1}^{n} p_{k}}\right)\right\}\right. \\
& =(1-(1-u), 1-(1-c))=(u, c)=s
\end{aligned}
$$

Thus we get,

$$
C F H W A\left(s_{1}, s_{2}, \ldots, s_{n}\right)=s
$$

(2). We have $\mathrm{s}_{\min }, \mathrm{s}_{\max }$ are the maximum and minimum FCNs, as $\mathrm{s}_{\min } \leq s_{i} \leq$ $s_{\max }$. Then,

is exist and now we have,

$$
\oplus_{i=1}^{n} s_{\min } p_{i} \leq \oplus_{i=1}^{n} s_{i} p_{i} \leq \oplus_{i=1}^{n} p_{i} s_{\max }
$$

$$
s_{\min } \leq \oplus_{i=1}^{n} s_{i} p_{i} \leq s_{\max }
$$

which implies that,

$$
s_{\min } \leq C F H W A\left(s_{1}, s_{2}, \ldots, s_{n}\right) \leq s_{\max }
$$

(3). If we have $s_{i} \leq s_{i}^{*}, \oplus_{i=1}^{n} s_{i} p_{i} \leq \oplus_{i=1}^{n} s_{i}^{*} p_{i}^{*}$.Then,

$$
C F H W A\left(s_{1}, s_{2}, \ldots, s_{n}\right) \leq C F H W A\left(s_{1}^{*}, s_{2}^{*}, \ldots, s_{n}^{*}\right)
$$

Which is the required prove.

\section{Credibility Fuzzy Hamacher Ordered Weighted Average AgGregation Operator}

In this subsection we developed CFHOWA operator by using Hamacher opertional laws and discus some fundamental properties. And in this subsection of the paper first we have to find all the score value or accuracy values of the given criteria and then we have to apply our proposed work to the given alternatives to check and find the best option.

Consider a collection of FCNs $\vartheta=\left\{s_{i}, i=1,2, \ldots, n\right\}$ having weight vector $w=$ $\left(w_{1}, w_{2}, \ldots, w_{n}\right)^{T}$. Then the mapping $\alpha^{n} \rightarrow \alpha_{j}$, is said to be CFHOWA operator. which is mathematically represented by the following equation.

$$
\begin{aligned}
& C F H O W A\left(s_{1}, s_{2}, \ldots, s_{n}\right)_{\sigma} \\
= & \left\{\oplus_{i=1}^{n} s_{i_{\sigma}} w_{i}\right\}
\end{aligned}
$$

In the next theorem we prove that the equation 6.1 is also a FCNs and the aggregated value of FCNs $\vartheta=\left\{s_{i}, i=1,2, \ldots, n\right\}$ is also a FCNs.

Theorem 3. Consider a collection of $\vartheta=\left\{s_{i}, i=1,2, \ldots, n\right\}$ is a FCNs having weight vector $w=\left(w_{1}, w_{2}, \ldots, w_{n}\right)^{T}$. So we can define CFHOWA operator as,

$$
\begin{aligned}
& C F H O W A\left(s_{1}, s_{2}, \ldots, s_{n}\right)_{\sigma} \\
= & \left\{\oplus_{i=1}^{n} s_{i_{\sigma}} w_{i}\right\} \\
= & \left\{\begin{array}{c}
\frac{\Pi_{i=1}^{n}\left(1+(\lambda-1) a_{\sigma i}\right)^{w_{i}}-\Pi_{i=1}^{n}\left(1-a_{\sigma i}\right)^{w_{i}}}{\Pi_{i=1}^{n}\left(1+(\lambda-1) a_{\sigma i}\right)^{w_{i}}+(\lambda-1) \Pi_{i=1}^{n}\left(1-a_{\sigma i}\right)^{w_{i}}}, \\
\frac{\lambda \Pi_{i=1}^{n} b_{i i}^{w_{i}}}{\Pi_{i=1}^{n}\left(1+(\lambda-1)\left(1-b_{\sigma i}\right)\right)^{w_{i}}+(\lambda-1) \Pi_{i=1}^{n} b_{\sigma i}^{w_{i}}}
\end{array}\right\}
\end{aligned}
$$


The maximum permutation values from the set of FCNs are denoted by $s_{i}$.

Proof. The proof of this theorem is like the previous one.

In the following theorem, some characteristics of CFHOWA were investigated and explained as under.

Theorem 4. Consider a collection of $\vartheta=\left\{s_{i}, i=1,2, \ldots, n\right\}$ is a FCNs having weight vector $w=\left(w_{1}, w_{2}, \ldots, w_{n}\right)^{T}$. Then we have some properties.

(1) (The idempotency): If $s_{i}=s=(u, c) \forall i=1, \ldots, n$. Then,

$$
C F H O W A\left(s_{1}, s_{2}, \ldots, s_{n}\right)=s
$$

(2) (Boundedness): If $s_{i}(i=1, \ldots, n)$, is a group of FCNs then a set $s_{\min }=$ $\left(\min _{i} u_{i}, \max _{i} c_{i}\right)$ and $s_{\max }=\left(\max _{i} u_{i}, \min _{i} c_{i}\right)$ are the maximum and minimum FCNs. Then,

$$
s_{\min } \leq C F H O W A\left(s_{1}, s_{2}, \ldots, s_{n}\right) \leq s_{\max }
$$

(3) (Monotonicity): Let $s_{i}(i=1, \ldots, n), s_{i}^{*}(i=1, \ldots, n)$ be the collection of FCNs such that $s_{i} \leq s_{i}^{*}$. Thus,

$$
\begin{array}{r}
\text { CFHOW }\left(s_{1}, s_{2}, \ldots, s_{n}\right) \\
\leq \quad C F H O W A\left(s_{1}^{*}, s_{2}^{*}, \ldots, s_{n}^{*}\right)
\end{array}
$$

Proof. The proof of this theorem is like the previous one.

\section{Credibility Fuzzy Hamacher Hybrid Weighted Average Aggregation Operator}

The CFHHWA operator evaluates a fuzzy reports value as well as its arranged condition. In this section of the paper, the CFHHWA and its basic properties were explained. Also the extra work is that in these proposed aggregation operators that we have to multiply a corresponding weight to a each of criteria or alternatives and then to apply our new aggregation operators to select the best option from a give set of option or alternatives.

Assume that $s_{i}(i=1, \ldots, n)$ is a set of FCNs in $\grave{U} \neq \phi$ having weight vector as $w=\left(w_{1}, w_{2}, \ldots, w_{n}\right)^{T}$, with $\sum_{i=1}^{n} w_{i}=1$ and $0 \leq w_{i} \leq 1$. Then the mapping $\alpha^{n} \rightarrow \alpha$,is said to be CFHHWA operator.

$$
\begin{aligned}
& C F H H W A\left(s_{1}, s_{2}, \ldots, s_{n}\right)^{\bullet} \\
= & \left(\oplus_{i=1}^{n} s_{i}^{\bullet} w_{i}\right)
\end{aligned}
$$

In the next theorem we prove that the equation 7.1 is also a FCN and also we discuss the hybrid weighted averaging operator and also the various cases about the weight and its conversion of various aggregation operators may be explained in detail. If we put the values of weight $w=\left(\frac{1}{n}, \frac{1}{n}, \ldots, \frac{1}{n}\right)^{T}$, The CFHHWA operator would become an CFHOWA operator.

Theorem 5. Consider a collection of $\vartheta=\left\{s_{i}, i=1,2, \ldots, n\right\}$ is a FCNs having weight vector and the associated weight $w=\left(w_{1}, w_{2}, \ldots, w_{n}\right)^{T}, \hat{w}=\left(\hat{w}_{1}, \hat{w}_{2}, \ldots, \hat{w}_{n}\right)^{T}$ 
respectively. As a result, the CFHHWA operator can be described as,

$$
\begin{aligned}
& C F H H W A\left(s_{1}, s_{2}, \ldots, s_{n}\right)^{\bullet} \\
= & \left\{\oplus_{i=1}^{n} s_{i}^{\bullet} w_{i}\right\} \\
= & \left\{\begin{array}{c}
\frac{\Pi_{i=1}^{n}\left(1+(\lambda-1) a_{i}^{\bullet}\right)^{w_{i}}-\Pi_{i=1}^{n}\left(1-a_{i}^{\bullet}\right)^{w_{i}}}{\Pi_{i=1}^{n}\left(1+(\lambda-1) a i^{\bullet}\right)_{i}+(\lambda-1) \Pi_{i=1}^{n}\left(1-a_{i}^{\bullet}\right)^{w_{i}}}, \\
\frac{\lambda \Pi_{i=1}^{n} b_{i}^{w_{i}}}{\Pi_{i=1}^{n}\left(1+(\lambda-1)\left(1-b_{i}^{\bullet}\right)\right)^{w_{i}}+(\lambda-1) \Pi_{i=1}^{n} b_{i}^{w_{\bullet}}}
\end{array}\right\}
\end{aligned}
$$

Where $s_{i}^{\bullet}$ represent the highest permutation values form the collection of FCNs . where $n$ show the balancing coefficient in $s_{i}^{\bullet}=n \hat{w}_{i} s_{i}^{\bullet}$.

Proof. The proof of this theorem is like the previous one.

In the next theorem we discuss some characteristic of the CFHHWA operator.

Theorem 6. Consider a collection of $\vartheta=\left\{s_{i}, i=1,2, \ldots, n\right\}$ is a FCNs having weight vector and the associated weight $w=\left(w_{1}, w_{2}, \ldots, w_{n}\right)^{T}, \hat{w}=\left(\hat{w}_{1}, \hat{w}_{2}, \ldots, \hat{w}_{n}\right)^{T}$ respectively. As a result, the CFHHWA operator can be described as, then we have some basic properties.

(1) (The idempotency): If $s_{i}=s=(u, c) \forall k=1, \ldots, n$. Then,

$$
\text { CFHHWA(s } \left.s_{1}, s_{2}, \ldots, s_{n}\right)^{\bullet}=s^{\bullet}
$$

(2) (Boundedness): If $s_{i}(i=1, \ldots, n)$ be the collection of FCNs so the set $s_{\min }=\left(\min _{i} u_{i}, \max _{i} c_{i}\right)$ and $s_{\max }=\left(\max _{i} u_{i}, \min _{i} c_{i}\right)$ are the maximum and minimum FCNs. Then,

$$
s_{\min }^{\bullet} \leq C F H H W A\left(s_{1}, s_{2}, \ldots, s_{n}\right)^{\bullet} \leq s_{\max }^{\bullet}
$$

(3) (Monotonicity): Let $s_{i}(i=1, \ldots, n), s_{i}^{*}(i=1, \ldots, n)$ be another collection of FCNs such that $s_{i} \leq s_{i}^{*}$. Thus,

$$
\begin{aligned}
& C F H H W A\left(s_{1}, s_{2}, \ldots, s_{n}\right)^{\bullet} \\
\leq & C F H H W A\left(s_{1}^{*}, s_{2}^{*}, \ldots, s_{n}^{*}\right)
\end{aligned}
$$

Proof. The proof of this theorem is like the previous one.

\section{Credibility Fuzzy Hamacher Weighted Geometric Aggregation \\ OPERATOR}

In this subsection we developed CFHWG operator by using Hamacher operational laws and discus some fundamental properties. Using the properties which is written as above (i) and (iv) we can proposed a new aggreagtion operator which is in a series way like CFHWG, CFHOWG and CFHHG.

Consider a collection of FCNs $\vartheta=\left\{s_{i}, i=1,2, \ldots, n\right\}$ having weight vector $w=\left(w_{1}, w_{2}, \ldots, w_{n}\right)^{T}$. Then the mapping $\vartheta^{n} \rightarrow \vartheta_{j}$, is said to be CFHWG operator. which is mathematically represented by the following equation.

$$
C F H W G\left(s_{1}, s_{2}, \ldots, s_{n}\right)=\otimes_{i=1}^{n} s_{i} \varsigma_{i}
$$


Theorem 7. Consider a collection of $\vartheta=\left\{s_{i}, i=1,2, \ldots, n\right\}$ is a FCNs having weight vector $w=\left(w_{1}, w_{2}, \ldots, w_{n}\right)^{T}$. Then we have a mapping $\alpha^{n} \rightarrow \alpha_{j}$, is said to be CFHWG operator.

$$
\begin{aligned}
& C F H W G\left(s_{1}, s_{2}, \ldots, s_{n}\right) \\
= & \left\{\otimes_{i=1}^{n} s_{i} w_{i}\right\}^{\varsigma_{i}} \\
= & \left\{\begin{array}{c}
\frac{\lambda \Pi_{i=1}^{n} a_{i}^{w_{i}}}{\Pi_{i=1}^{n}\left(1+(\lambda-1)\left(1-a_{i}\right)\right)^{w_{i}}+(\lambda-1) \Pi_{i=1}^{n} a_{i}^{w_{i}}}, \\
\frac{\prod_{i=1}^{n}\left(1+(\lambda-1) b_{i}\right)_{i}-\Pi_{i=1}^{n}\left(1-b_{i}\right)^{w_{i}}}{\prod_{i=1}^{n}\left(1+(\lambda-1) b_{i}\right)^{w_{i}}+(\lambda-1) \Pi_{i=1}^{n}\left(1-b_{i}\right)^{w_{i}}}
\end{array}\right\}
\end{aligned}
$$

Proof. The proof is same as above.

We must investigate it on the support of a brief analysis, assuming that $s_{i}$ is FCNs. So, by Definition, $\left\{\otimes_{i=1}^{n} s_{i} w_{i}\right\}^{\varsigma_{i}}$ and is also FCNs. Therefore, CFHWG $\left(s_{1}, s_{2}, \ldots, s_{n}\right)$ is also an CFHWG under fuzzy credibility environment.

In the next theorem we discuss some characteristic of proposed CFHWG operator.

Theorem 8. Consider a collection of $\vartheta=\left\{s_{i}, i=1,2, \ldots, n\right\}$ is a FCNs having weight vector $w=\left(w_{1}, w_{2}, \ldots, w_{n}\right)^{T}$. Then we defined some properties as follows.

(1) (Idempotency): If $s_{i}=s=(u, c) \forall i=1, \ldots, n$. Then,

$$
C F H W G\left(s_{1}, s_{2}, \ldots, s_{n}\right)=s
$$

(2) (Boundedness): If $s_{i}(i=1, \ldots, n)^{-}$be the collection of FCNs so the set $s_{\min }=\left(\min _{i} u_{i}, \min _{i} c_{i}\right)$ and $s_{\max }=\left(\max _{i} u_{i}, \max _{i} c_{i}\right)$ are the maximum and minimum FCNs. Then,

$$
s_{\min } \leq C F H W G\left(s_{1}, s_{2}, \ldots, s_{n}\right) \leq s_{\max }
$$

(3) (Monotonicity): Let $s_{i}(i=1, \ldots, n), s_{i}^{*}(i=1, \ldots, n)$ are the collection of FCNs implies that $s_{i} \leq s_{i}^{*}$. Thus,

$$
\begin{array}{r}
C F H W G\left(s_{1}, s_{2}, \ldots, s_{n}\right) \\
\leq \quad C F H W G\left(s_{1}^{*}, s_{2}^{*}, \ldots, s_{n}^{*}\right)
\end{array}
$$

Proof. The proof is same as above.

\section{Credibility Fuzzy Hamacher Ordered Weighted Geometric OPERATOR}

In this subsection we develop CFHOWG operator by using Hamacher operational laws and discus some fundamental properties. In this we have to find a score and accuracy function and then we have to apply our proposed work (aggregation operators) to select the best option from a set of options.

Consider a collection of FCNs $\vartheta=\left\{s_{i}, i=1,2, \ldots, n\right\}$ having weight vector $w=$ $\left(w_{1}, w_{2}, \ldots, w_{n}\right)^{T}$. Then the mapping $\alpha^{n} \rightarrow \alpha_{j}$, is said to be CFHOWG operator. which is mathematically represented by the following equation.

$$
\begin{aligned}
& C F H O W G\left(s_{1}, s_{2}, \ldots, s_{n}\right)_{\sigma} \\
= & \left\{\otimes_{i=1}^{n} s_{i_{\sigma}} w_{i}\right\}
\end{aligned}
$$

In the next theorem we prove that the equation 9.1 is also a $\mathrm{FCN}$ and the aggregated value of FCNs $\vartheta=\left\{s_{i}, i=1,2, \ldots, n\right\}$ is also a FCNs. 
Theorem 9. Consider a collection of $\vartheta=\left\{s_{i}, i=1,2, \ldots, n\right\}$ is a FCNs having weight vector $w=\left(w_{1}, w_{2}, \ldots, w_{n}\right)^{T}$. So CFHOWG operator is written as,

$$
\begin{aligned}
& \text { CFHOWG }\left(s_{1}, s_{2}, \ldots, s_{n}\right)_{\sigma} \\
& =\left\{\otimes_{i=1}^{n} s_{i_{\sigma}} w_{i}\right\} \\
& =\left\{\begin{array}{c}
\lambda \Pi_{i=1}^{n} a_{\sigma i}^{w_{i}} \\
\frac{\Pi_{i=1}^{n}\left(1+(\lambda-1)\left(1-a_{\sigma i}\right) w^{w_{i}}+(\lambda-1) \Pi_{i=1}^{n} a_{\sigma i}^{w_{i}}\right.}{\bar{\Pi}_{i=1}^{n}\left(1+(\lambda-1) b_{\sigma i}\right)^{w_{i}}-\Pi_{i=1}^{n}\left(1-b_{\sigma i}\right)^{w_{i}}} \\
\overline{\Pi_{i=1}^{n}\left(1+(\lambda-1) b_{\sigma i}\right)^{w_{i}}+(\lambda-1) \Pi_{i=1}^{n}\left(1-b_{\sigma i}\right)^{w_{i}}}
\end{array}\right\}
\end{aligned}
$$

$\left\{\otimes_{i=1}^{n} s_{i_{\sigma}} w_{i}\right\}$ show the largest number of permutation for the collection of FCNs.

Proof. The proof of this theorem is like the previous one.

In the next theorem we discuss some characteristic of FCHOWG operators.

Theorem 10. Consider a collection $s_{i}(i=1, \ldots, n)$ of FCNs and the weight vector is denoted by $w=\left(w_{1}, w_{2}, \ldots, w_{n}\right)^{T}$, with $\sum_{i=1}^{n} w_{i}=1$ and $0 \leq w_{i} \leq 1$.

(1) (The idempotency): If $s_{i}=s=(u, c) \forall k=1, \ldots, n$. Then,

$$
\text { FCHOWG }\left(s_{1}, s_{2}, \ldots, s_{n}\right)=s
$$

(2) (Boundedness): If $s_{i}(i=1, \ldots, n)^{-}$be the collection of FCNs then a set $s_{\min }=\left(\min _{i} u_{i}, \min _{i} c_{i}\right)$ and $s_{\max }=\left(\max _{i} u_{i}, \max _{i} c_{i}\right)$ are the maximum and minimum FCNs. Then,

$$
s_{\min } \leq C F H O W G\left(s_{1}, s_{2}, \ldots, s_{n}\right) \leq s_{\max }
$$

(3) (Monotonicity): Let $s_{i}(i=1, \ldots, n), s_{i}^{*}(i=1, \ldots, n)$ are the family of FCNs implies that $s_{i} \leq s_{i}^{*}$. Thus,

$$
\begin{array}{r}
C F H O W G\left(s_{1}, s_{2}, \ldots, s_{n}\right) \\
\leq \quad C F H O W G\left(s_{1}^{*}, s_{2}^{*}, \ldots, s_{n}^{*}\right)
\end{array}
$$

Proof. The proof of this theorem is like the previous one.

\section{Credibility Fuzzy Hamacher Hybrid Geometric Aggregation OPERATOR}

In this section we can explained the basic properties of developed aggregation operators especially CFHHG. On the basic operational laws of Hamacher t-norm and Hamacher t-conorm we can explained the CFHHG operators. And here firstly an associated weight vectors is multiply to the given alternatives or criteria and then our new aggregation is applied to select the best option.

Let $s_{i}(i=1, \ldots, n)$ be the collection of FCNs and the weight vector and associated vector is denoted by $w=\left(w_{1}, w_{2}, \ldots, w_{n}\right)^{T}$, such that $\sum_{i=1}^{n} w_{i}=1$ and $0 \leq w_{i} \leq$ $1, \hat{w}=\left(\hat{w}_{1}, \hat{w}_{2}, \ldots, \hat{w}_{n}\right)^{T}$, with $\sum_{i=1}^{n} \hat{w}_{i}=1$ and $0 \leq \hat{w}_{i} \leq 1$ respectively then we can write CFHHG operator as,

$$
\begin{aligned}
& C F H H G\left(s_{1}, s_{2}, \ldots, s_{n}\right)^{\bullet} \\
= & \left\{\oplus_{i=1}^{n} s_{i}^{\bullet} w_{i}\right\} \\
= & \left\{\begin{array}{c}
\frac{\lambda \Pi_{i=1}^{n} a_{i \bullet}^{w_{i}}}{\prod_{i=1}^{n}\left(1+(\lambda-1)\left(1-a_{i}^{\bullet}\right)\right)^{w_{i}}+(\lambda-1) \Pi_{i=1}^{n} w_{i}^{w_{i}}}, \\
\Pi_{i=1}^{n}\left(1+(\lambda-1) b_{i}^{\bullet}\right)^{w_{i}}-\Pi_{i=1}^{n}\left(1-b_{i}^{\bullet}\right)_{i}^{w_{i}} \\
\prod_{i=1}^{n}\left(1+(\lambda-1) b_{i}^{\bullet}\right)^{w_{i}}+(\lambda-1) \Pi_{i=1}^{n}\left(1-b_{i}^{\bullet}\right)^{w_{i}}
\end{array}\right\}
\end{aligned}
$$


In the next theorem we prove that the equation 10.1 is also a $\mathrm{FCN}$ and the aggregated value of FCNs $\vartheta=\left\{s_{i}, i=1,2, \ldots, n\right\}$ is also a FCNs.

Theorem 11. Let $s_{i}(i=1, \ldots, n)$ be the collection of FCNs and the weight vector is denoted by $w=\left(w_{1}, w_{2}, \ldots, w_{n}\right)^{T}$, such that $\sum_{i=1}^{n} w_{i}=1$ and $0 \leq w_{i} \leq 1$. Let $\hat{w}=\left(\hat{w}_{1}, \hat{w}_{2}, \ldots, \hat{w}_{n}\right)^{T}$, with $\sum_{i=1}^{n} \hat{w}_{i}=1$ and $0 \leq \hat{w}_{i} \leq 1$ having associated weight then we can write CFHHG operator as,

$$
\begin{aligned}
& C F H H G\left(s_{1}, s_{2}, \ldots, s_{n}\right) \\
= & \left\{\oplus_{i=1}^{n} s_{i}^{\bullet} \varsigma_{i}\right\} \\
= & \left\{\begin{array}{c}
\frac{\lambda \Pi_{i=1}^{n} a_{i \bullet}^{w_{i}}}{\Pi_{i=1}^{n}\left(1+(\lambda-1)\left(1-a_{i}^{\bullet}\right)\right)^{w_{i}}+(\lambda-1) \Pi_{i=1}^{n} a_{i}^{w_{i}}}, \\
\frac{\Pi_{i=1}^{n}\left(1+(\lambda-1) b_{i}^{\bullet}\right)^{w_{i}}-\Pi_{i=1}^{n}\left(1-b_{i}^{\bullet}\right)_{i}^{\omega_{i}}}{\Pi_{i=1}^{n}\left(1+(\lambda-1) b_{i}^{\bullet}\right)^{w_{i}}+(\lambda-1) \Pi_{i=1}^{n}\left(1-b_{i}^{\bullet}\right)^{w_{i}}}
\end{array}\right\}
\end{aligned}
$$

Where $s_{i}^{\bullet}$ denotes the highest permutation values from the collection of FCNs. where $n$ show the balancing coefficient.

$$
s_{i}^{\bullet}=n \hat{w}_{i} s_{i}^{\bullet}
$$

Proof. The proof is same as above.

The special cases of various aggregation operators were discuss by having different values of weights. So when we used the weight as as special case like $w=\left(\frac{1}{n}, \frac{1}{n}, \ldots, \frac{1}{n}\right)^{T}$ then the CFHHG is converted in to the form of CFHOWG operators.

Theorem 12. Let $s_{i}(i=1, \ldots, n)$ be the collection of FCNs having associated weight $\hat{w}=\left(\hat{w}_{1}, \hat{w}_{2}, \ldots, \hat{w}_{n}\right)^{T}$, such that $\sum_{i=1}^{n} \hat{w}_{i}=1$ and $0 \leq \hat{w}_{i} \leq 1$. Consider the weight vector for FCNs is denoted by $w=\left(w_{1}, w_{2}, \ldots, w_{n}\right)^{T}$, with $\sum_{i=1}^{n} w_{i}=1$ and $0 \leq w_{i} \leq 1$. Then we define CFHHG operator as,

(1) (Idempotency): If $s_{i}=s=(t, c) \forall k=1, \ldots, n$. Then,

$$
C F H H G\left(s_{1}, s_{2}, \ldots, s_{n}\right)^{\bullet}=s^{\bullet}
$$

(2) (Boundedness): If $s_{i}(i=1, \ldots, n)$ be the collection of FCNs then a set $s_{\min }=\left(\min _{i} u_{i}, \min _{i} c_{i}\right)$ and $s_{\max }=\left(\max _{i} u_{i}, \max _{i} c_{i}\right)$ are the maximum and minimum FCNs. Then,

$$
s_{\min }^{\bullet} \leq C F H H G\left(s_{1}, s_{2}, \ldots, s_{n}\right)^{\bullet} \leq s_{\max }^{\bullet}
$$

(3) (Monotonicity): Let $s_{i}(i=1, \ldots, n), s_{i}^{*}(i=1, \ldots, n)$ are the family of FCNs implies that $s_{i} \leq s_{i}^{*}$. Thus,

$$
\begin{aligned}
& C F H H G\left(s_{1}, s_{2}, \ldots, s_{n}\right)^{\bullet} \\
\leq & C F H H G\left(s_{1}^{*}, s_{2}^{*}, \ldots, s_{n}^{*}\right)
\end{aligned}
$$

Proof. The proof of this theorem is like the previous one.

\section{MCGDM Problems On Credibility Fuzzy Hamacher Aggregation OPERATORS}

As the social atmosphere warms, the importance of DM issues grows in this real - world situation. As a result, in this situation, an expert's capacity to make a reasonable and knowledgeable conclusion is disturbed. In real-world situations, group decision-making systems rely on the feedback of a group of trained specialists 
to arrive at an effective solution. As a result, MCGDM has a defined ability and regulatory structure to improve and assess various competing criteria in all areas of DMs in order to achieve the most effective and practical DMs outcomes. We also explored and suggested an Extended GRA-method and TODIM methodology using FCNs, in which the experts introduced their FCNs provides strategic. When using the established model with CFHWA/CFHWG aggregation operators, we must discuss the various phases.

Assume that $m$ is represented by a set of alternatives, and that $m=\left\{p_{1}, p_{2}, \ldots, p_{m}\right\}$ defines a set of decision alternatives respectively. The alternatives and criteria were represented by $P_{i}(i=1,2, \ldots, m), c_{j}(j=1,2, \ldots, n)$ which is used in the proposed techniques also there is also a group decision maker and the decision matrix having data in the form of FCNs is denoted by $D=\left\{D_{1}, D_{2}, \ldots, D_{t}\right\}$. So after that we have to find the best option from a given possible option having weight vector which play an important rule in this process. The further description of the proposed algorithm is as under.

11.1. Algorithm-I. In the algorithm-I we can explained some steps which is apply for further selection of best alternatives. And also the step is described and calculated as follows.

\subsection{Representation Of Data.}

Step-1: The first step of the proposed method is that we have represent the data in the form of matrix shape having alternatives and criteria. which will be written as.

$$
\begin{gathered}
M=\left[\tau\left(k_{i j}^{l}\right)\right]_{m \times n} \\
\begin{array}{c}
\text { Alternatives } \\
A_{1} \\
A_{2} \\
\vdots \\
A_{n}
\end{array} \quad\left[\begin{array}{llllll}
c_{1} & c_{2} & c_{3} & \cdots & c_{m} \\
k_{11}^{l} & k_{12}^{l} & k_{13}^{l} & \cdots & k_{1 m}^{l} \\
k_{21}^{l} & k_{22}^{l} & k_{23}^{l} & \cdots & k_{2 m}^{l} \\
\vdots & \vdots & \vdots & \ddots & \vdots \\
k_{n 1}^{l} & k_{n 2}^{l} & k_{n 3}^{l} & \cdots & k_{n m}^{l}
\end{array}\right]
\end{gathered}
$$

Here using the FCNs also the decision maker is denoted by $D_{i}$. And also $\left(k_{i j}^{l}\right)$ represent the DM values having alternatives and criteria.

\subsection{Representation of Aggregated Matrix.}

Step-2: The second step is about to aggregate all the DM, which will give us a collective DM. For the aggregation of all DM using the proposed aggregation operators having its corresponding weights.

$$
M=\left[\tau\left(k_{i j}\right)\right]_{(m \times n)^{\prime}}
$$

and using these proposed aggregation operators to collect all the decision matrix into one single decision matrix.

$$
\begin{aligned}
& C F H W G\left(s_{1}, s_{2}, \ldots, s_{n}\right) \\
= & \left\{\otimes_{i=1}^{n} s_{i} w_{i}\right\}^{\varsigma_{i}} \\
= & \left\{\begin{array}{c}
\frac{\lambda \Pi_{i=1}^{n} a_{i}^{w_{i}}}{\prod_{i=1}^{n}\left(1+(\lambda-1)\left(1-a_{i}\right)\right)^{w_{i}}+(\lambda-1) \Pi_{i=1}^{n} a_{i}^{w_{i}}}, \\
\overline{\Pi_{i=1}^{n}\left(1+(\lambda-1) b_{i}\right)_{i}-\Pi_{i=1}^{n}\left(1-b_{i}\right)_{i}^{w_{i}}} \\
\overline{\prod_{i=1}^{n}\left(1+(\lambda-1) b_{i}\right)^{w_{i}}+(\lambda-1) \Pi_{i=1}^{n}\left(1-b_{i}\right)^{w_{i}}}
\end{array}\right\}
\end{aligned}
$$


Or

$$
\begin{aligned}
& C F H W A\left(s_{1}, s_{2}, \ldots, s_{n}\right) \\
= & \left\{\oplus_{i=1}^{n} s_{i} w_{i}\right\} \\
= & \left\{\begin{array}{c}
\frac{\Pi_{i=1}^{n}\left(1+(\lambda-1) a_{i}\right)^{w_{i}}-\Pi_{i=1}^{n}\left(1-a_{i}\right)^{w_{i}}}{\Pi_{i=1}^{n}\left(1+(\lambda-1) a_{i}\right)^{w_{i}}+(\lambda-1) \Pi_{i=1}^{n}\left(1-a_{i}\right)^{w_{i}}} \\
\frac{\lambda \Pi_{i=1}^{n} b_{i}^{w_{i}}}{\Pi_{i=1}^{n}\left(1+(\lambda-1)\left(1-b_{i}\right)\right)^{w_{i}}+(\lambda-1) \Pi_{i=1}^{n} b_{i}^{w_{i}}}
\end{array}\right\}
\end{aligned}
$$

\subsection{Determination of score function and Ranking Results.}

Step-3: The third step of the proposed method is to find the score function and due to the score function we have to rank all the alternatives to get the best options. Further higher values of the score function will arranged as first and do it for all the other alternatives to get the required results.

\subsection{Flow Chart For Algorithm-I}

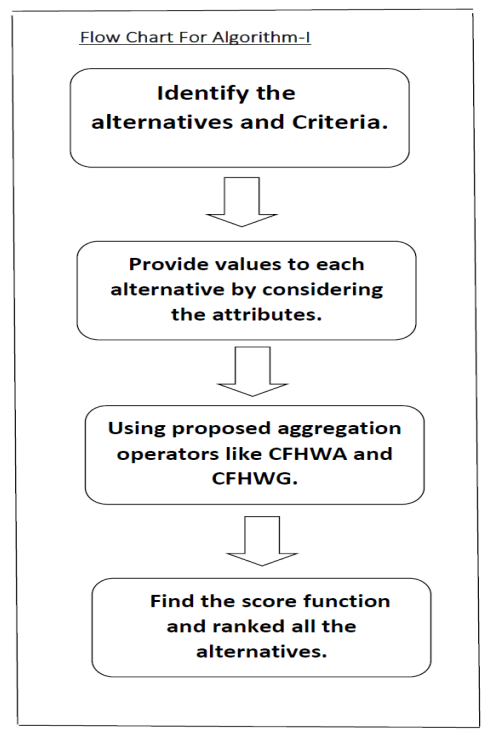

11.6. Algorithm II. In this subsection we have proposed a second algorithm-II in which there is some steps which is discus as under. On the basis of Hamacher tnorm and t-conorm we have introduce various aggregation operators like CFHWA, CFHOWA, CFHHWA, CFHWG, CFHOWG and CFHHG. And further we have apply this aggregation operators to solve MCGDM issue to select the best option from a given data. Further the detail description of this algorithm were explained as follows.

\subsection{Representation Of Data.}

Step-1: The first step of the proposed method is that we have represent the data in the form of matrix shape having alternatives and criteria. which will be written as.

$$
M=\left[\tau\left(k_{i j}^{l}\right)\right]_{m \times n}
$$


Here using the FCNs also the decision maker is denoted by $D_{i}$. And also $\left(k_{i j}^{l}\right)$ represent the DMs fuzzy credibility values having alternatives and criteria.

$$
D=\begin{array}{lllll}
\text { Alternatives } \\
A_{1} \\
A_{2} \\
\vdots \\
A_{n}
\end{array} \quad\left[\begin{array}{lllll}
c_{1} & c_{2} & c_{3} & \cdots & c_{m} \\
k_{11}^{l} & k_{12}^{l} & k_{13}^{l} & \cdots & k_{1 m}^{l} \\
k_{21}^{l} & k_{22}^{l} & k_{23}^{l} & \cdots & k_{2 m}^{l} \\
\vdots & \vdots & \vdots & \ddots & \vdots \\
k_{n 1}^{l} & k_{n 2}^{l} & k_{n 3}^{l} & \cdots & k_{n m}^{l}
\end{array}\right]
$$

\subsection{Representation of Aggregated Matrix.}

Step-2: The second step is about to aggregate all the DM, which will give us a collective DM. For the aggregation of all DM using the proposed aggregation operators having its corresponding weights.

$$
\begin{aligned}
& C F H W G\left(s_{1}, s_{2}, \ldots, s_{n}\right) \\
= & \left\{\otimes_{i=1}^{n} s_{i} w_{i}\right\}^{\varsigma_{i}} \\
= & \left\{\begin{array}{c}
\frac{\lambda \Pi_{i=1}^{n} a_{i}^{w_{i}}}{\prod_{i=1}^{n}\left(1+(\lambda-1)\left(1-a_{i}\right)\right)^{w_{i}}+(\lambda-1) \Pi_{i=1}^{n} a_{i}^{w_{i}}}, \\
\frac{\Pi_{i=1}^{n}\left(1+(\lambda-1) b_{i}\right)^{w_{i}}-\Pi_{i=1}^{n}\left(1-b_{i}\right)^{w_{i}}}{\Pi_{i=1}^{n}\left(1+(\lambda-1) b_{i}\right)^{w_{i}}+(\lambda-1) \Pi_{i=1}^{n}\left(1-b_{i}\right)^{w_{i}}}
\end{array}\right\}
\end{aligned}
$$

Or

$$
\begin{aligned}
& C F H W A\left(s_{1}, s_{2}, \ldots, s_{n}\right) \\
= & \left\{\oplus_{i=1}^{n} s_{i} w_{i}\right\} \\
= & \left\{\begin{array}{c}
\frac{\Pi_{i=1}^{n}\left(1+(\lambda-1) a_{i}\right)^{w_{i}}-\Pi_{i=1}^{n}\left(1-a_{i}\right)^{w_{i}}}{\Pi_{i=1}^{n}\left(1+(\lambda-1) a_{i}\right)^{w_{i}}+(\lambda-1) \Pi_{i=1}^{n}\left(1-a_{i}\right)^{w_{i}}} \\
\frac{\lambda \Pi_{i=1}^{n} b_{i}^{w_{i}}}{\Pi_{i=1}^{n}\left(1+(\lambda-1)\left(1-b_{i}\right)\right)^{w_{i}}+(\lambda-1) \Pi_{i=1}^{n} b_{i}^{w_{i}}}
\end{array}\right\}
\end{aligned}
$$

\subsection{Determination Of PIS and NIS.}

Step -3: Find the similarity measured from FCNs-PIS (solution of fuzzy credibility positive ideal solution) and FCNs-NIS (solution of fuzzy credibility negative ideal solution). In order to find the similarity measure between each alternatives we have to use the below equations.

$$
\begin{aligned}
& \operatorname{PIS}\left\{\vartheta=\left\{s_{i}, i=1,2, \ldots, n\right\}\right\}=\max _{i}\left(u_{a}\left(x_{i}\right), v_{a}\left(x_{i}\right)\right) \\
& \operatorname{NIS}\left\{\vartheta=\left\{s_{i}, i=1,2, \ldots, n\right\}\right\}=\min _{i}\left(u_{a}\left(x_{i}\right), v_{a}\left(x_{i}\right)\right) \\
& S(A, B)=1-\frac{1}{2 n} \sum_{i=1}^{n}\left|s_{a}\left(x_{i}\right)-s_{b}\left(x_{i}\right)\right| \\
& O R \\
& S(A, B)=1-\frac{1}{2 n} \sum_{i=1}^{n}\left|\left(u_{a}\left(x_{i}\right)-v_{a}\left(x_{i}\right)\right)-\left(u_{b}\left(x_{i}\right)-v_{b}\left(x_{i}\right)\right)\right|
\end{aligned}
$$

where $\mathrm{a}$ and $\mathrm{b}$ are the FCNs then $s_{a}\left(x_{i}\right)=u_{a}\left(x_{i}\right)-v_{a}\left(x_{i}\right)$ and $s_{b}\left(x_{i}\right)=u_{b}\left(x_{i}\right)-$ $v_{b}\left(x_{i}\right)$ is also the FCNs. 


\subsection{Determination of Grey Relational Coefficient.}

Step -4: Find the Grey relational coefficient of alternatives. We have to use the following formula to find the Grey relational coefficient between each alternative and FCNs-PIS.

$$
V_{i j}^{+}=V\left(B_{j}^{+}, B_{i j}\right)=\frac{\min _{i} \min _{j} S\left(B_{j}^{+}, B_{i j}\right)+\rho \max _{i} \max _{j} S\left(B_{j}^{+}, B_{i j}\right)}{S\left(B_{j}^{+}, B_{i j}\right)+\rho \max _{i} \max _{j} S\left(B_{j}^{+}, B_{i j}\right)}
$$

where $\rho$ is the recognition coefficient $\rho \in[0,1]$, let $\rho=0.5$ and $i=1,2, \ldots, m$ and $j=1,2, \ldots, n$.

We have to use the following formula to find the Grey relational coefficient between each Alternative and FCNs-NIS.

$$
V_{i j}^{-}=V\left(B_{j}^{-}, B_{i j}\right)=\frac{\min _{i} \min _{j} S\left(B_{j}^{-}, B_{i j}\right)+\rho \max _{i} \max _{j} S\left(B_{j}^{-}, B_{i j}\right)}{S\left(B_{j}^{-}, B_{i j}\right)+\rho \max _{i} \max _{j} S\left(B_{j}^{-}, B_{i j}\right)}
$$

\subsection{Determination Of Grey Relational Grade.}

Step -5: Find the Grey relational grade of alternatives.

$$
\begin{aligned}
& u_{i}^{+}=\sum_{j=1}^{n} V_{i j}^{+} \times w_{j} \text { and } \sum_{j=1}^{n} w_{j}=1 \\
& u_{i}^{-}=\sum_{j=1}^{n} V_{i j}^{-} \times w_{j} \text { and } \sum_{j=1}^{n} w_{j}=1
\end{aligned}
$$

\subsection{Determination Of Relative Closeness Coefficient.}

Step -6: Find the relative Grey relational grade with regards to FCNs-PIS. The Relative grey relational grade of an alternative $A_{i}$ with regard to the fuzzy credibility positive-ideal solution (FCNs-PIS) $A^{+}$can be find as:

$$
S I_{i}=\frac{S I_{i}^{+}}{S I_{i}^{+}+S I_{i}^{-}}
$$

\subsection{Ranking Of alternatives.}

Step -7: Sorting the alternatives.

After finding the relative grey relational grade of each alternatives are listed in descending order of $c I_{(s)}$. The larger value relative grey relational grade of some alternative represents that it is close to FCNs-PIS. As a result the most appropriate choice should be the alternatives with the higher value of closeness.

\subsection{Ending of Algorithm.}

Step -8: This is last step of proposed method in which the final result will be shown. And where all the ranking position is shown. 


\subsection{Flow Chart For Algorithm-II}

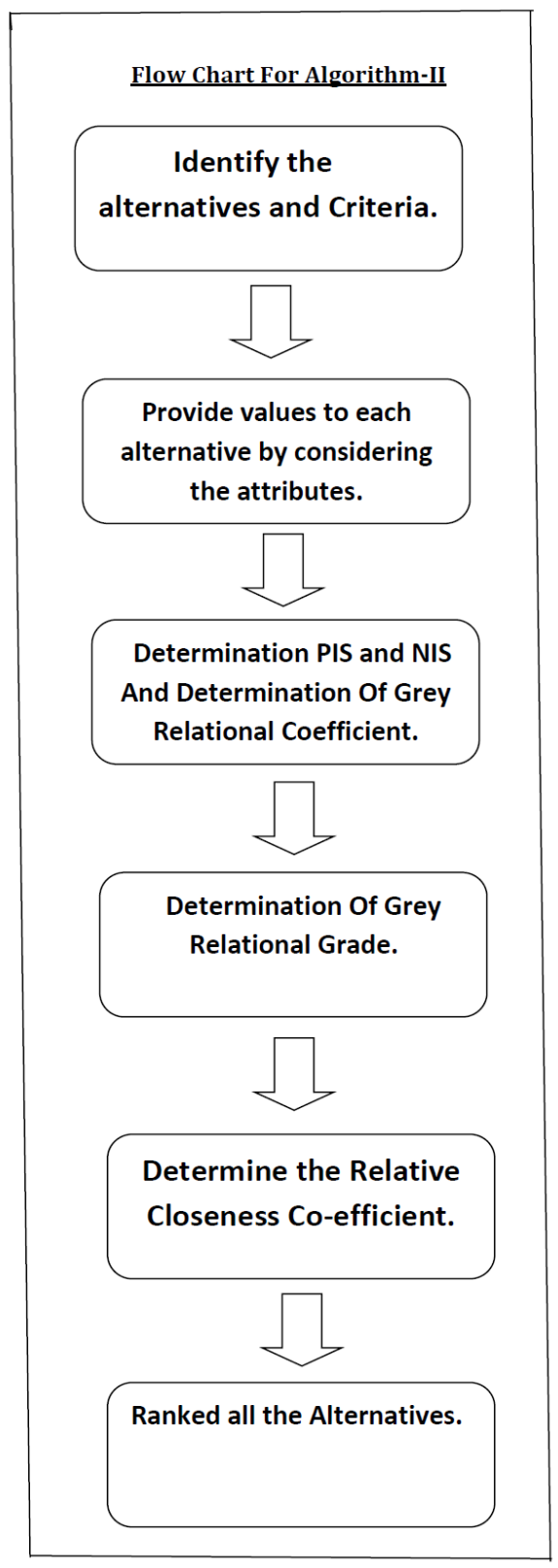

11.16. Case study. The economic development of the country is effected by the energy crises. Among the various energy sources, electricity is the most important energy source that is in popular by various economic sectors. There are many electricity crises which is faced at different condition or level in Pakistan. There are many issues which is created by the electricity shortage as well as affect the economy level of Pakistan. The absence of planning and policy formulation is 
wholly responsible for this electricity shortage. By providing a good policy and having a best planning by the government to improve the electricity demands. So the various sources and the factor affecting these sources are discussed further. For this we have to define and discussed our step wise approach to select the best option for energy crises. Further we have a set of four alternatives $\left\{A_{1}, A_{2}, A_{3}, A_{4}\right\}$ which is the causes of electricity and the description of these alternatives is as,

$A_{1}$ is For Hydro Power,

$\mathrm{A}_{2}$ is For Solar Energy,

$\mathrm{A}_{3}$ is For Fuel Energy,

$\mathrm{A}_{4}$ is Wind Energy.

Also the detail of criteria is as follows and denoted by $\left\{c_{1}, c_{2}, c_{3}, c_{4}, c_{5}\right\}$.

$c_{1}$ is For Environmental Factor,

$\mathrm{c}_{2}$ is For Economic Factor,

$c_{3}$ is For Technical Factor,

$\mathrm{c}_{4}$ is For Socio-political Factor,

$c_{5}$ is For Mechanical Factor.

11.17. Proposed Methodology. To apply and verify our own proposed methods we have taken a collection of three DMs which is denoted as $\left(\mathrm{DM}_{1}, \mathrm{DM}_{2}, \mathrm{DM}_{3}\right)$ having weight vectors $w=(0.2,0.3,0.5)^{T}$. However we have use these weight to aggregate these DMs into a collective matrix. Further also we have criteria weight vector which is denoted by $\varsigma=(0.21,0.24,0.22,0.15,0.18)^{T}$ while using proposed aggregation operators. And also to choose the good option from all the other alternatives so we will used score and accuracy function respectively. Further the detail of alternatives and criteria were explained as above and here we have to use only the proposed aggregation operators like CFHWA/CFHWG to get the best results.

11.18. Illustrative Example Based on Algorithm-I. Here the steps of proposed method were apply as on the numerical example which is discus as under. The detail of alternatives, criteria and weight vectors were discussed in detail. Also the steps is calculated and the results is tabulated as under. But here we can take the data in the form of FCNs and the developed aggregation operators like CFHWA/CFHWG operators were apply to get the best results. Also the steps of algorithm-I were find as under.

Step-1: The first step of the proposed method is that we have to represent the data in the form of matrix shape having alternatives and criteria in the form of FCNs. Here the $M=\left[\tau\left(k_{i j}^{l}\right)\right]_{(m \times n)}$ matrix form is presented as by Table-1, Table-2 and Table-3.

Table-1: FCNs information by $D_{1}$

\begin{tabular}{l|l|l|l|r|r|}
\hline \hline Alternatives & \multicolumn{1}{l}{$c_{1}$} & $c_{2}$ & $c_{3}$ & $c_{4}$ & $c_{5}$ \\
\hline \hline$p_{1}$ & $(0.8,0.1)$ & $(0.5,0.4)$ & $(0.2,0.5)$ & $(0.5,0.4)$ & $(0.2,0.7)$ \\
$p_{2}$ & $(0.1,0.8)$ & $(0.6,0.2)$ & $(0.1,0.9)$ & $(0.4,0.3)$ & $(0.2,0.6)$ \\
$p_{3}$ & $(0.6,0.1)$ & $(0.2,0.6)$, & $(0.1,0.9)$ & $(0.7,0.1)$ & $(0.4,0.3)$ \\
$p_{4}$ & $(0.7,0.2)$ & $(0.6,0.3)$ & $(0.6,0.2)$ & $(0.1,0.8)$ & $(0.5,0.2)$ \\
\hline
\end{tabular}


Table-2: FCNs information by $D_{2}$

\begin{tabular}{l|l|l|r|l|r}
\hline \hline Alternatives & $c_{1}$ & $c_{2}$ & $c_{3}$ & $c_{4}$ & $c_{5}$ \\
\hline \hline$p_{1}$ & $(0.2,0.5)$ & $(0.5,0.4)$ & $(0.8,0.1)$ & $(0.5,0.4)$ & $(0.2,0.5)$ \\
$p_{2}$ & $(0.1,0.9)$ & $(0.6,0.2)$ & $(0.1,0.8)$ & $(0.6,0.2)$ & $(0.1,0.9)$ \\
$p_{3}$ & $(0.1,0.9)$ & $(0.2,0.6)$, & $(0.6,0.1)$ & $(0.2,0.6)$, & $(0.1,0.9)$ \\
$p_{4}$ & $(0.6,0.2)$ & $(0.6,0.3)$ & $(0.7,0.2)$ & $(0.6,0.3)$ & $(0.6,0.2)$ \\
\hline
\end{tabular}

Table-3: FCNs information by $D_{3}$

\begin{tabular}{l|r|r|r|l|r}
\hline \hline Alternatives & $c_{1}$ & $c_{2}$ & $c_{3}$ & $c_{4}$ & $c_{5}$ \\
\hline \hline$p_{1}$ & $(0.8,0.1)$ & $(0.5,0.4)$ & $(0.2,0.7)$ & $(0.5,0.4)$ & $(0.2,0.5)$ \\
$p_{2}$ & $(0.1,0.8)$ & $(0.4,0.3)$ & $(0.2,0.6)$ & $(0.6,0.2)$ & $(0.1,0.9)$ \\
$p_{3}$ & $(0.6,0.1)$ & $(0.7,0.1)$ & $(0.4,0.3)$ & $(0.2,0.6)$, & $(0.1,0.9)$ \\
$p_{4}$ & $(0.7,0.2)$ & $(0.1,0.8)$ & $(0.5,0.2)$ & $(0.6,0.3)$ & $(0.6,0.2)$ \\
\hline
\end{tabular}

Step -2: The second step is about to aggregate all the DM, which will give us a collective DM using the developed aggregation operators CFHWA/CFHWG which is presented in Table- 4 .

Table-4: The aggregated matrix using proposed aggregation operators.

\begin{tabular}{l|l|l|l|l|l}
\hline \hline Alternatives & \multicolumn{1}{c|}{$c_{1}$} & \multicolumn{1}{c|}{$c_{2}$} & \multicolumn{1}{c|}{$c_{3}$} & \multicolumn{1}{c|}{$c_{4}$} & $c_{5}$ \\
\hline \hline$p_{1}$ & $(0.08,0.01)$ & $(0.06,0.02)$ & $(0.01,0.02)$ & $(0.06,0.02)$ & $(0.003,0.03)$ \\
$p_{2}$ & $(0.0004,0.05)$ & $(0.12,0.01)$ & $(0.0004,0.04)$ & $(0.04,0.01)$ & $(0.001,0.01)$ \\
$p_{3}$ & $(0.003,0.30)$ & $(0.003,0.03)$ & $(0.002,0.01)$ & $(0.06,0.02)$ & $(0.07,0.008)$ \\
$p_{4}$ & $(0.18,0.012)$ & $(0.13,0.02)$ & $(0.14,0.012)$ & $(0.002,0.02)$ & $(0.08,0.01)$ \\
\hline
\end{tabular}

Step-3: The score values of each alternatives is $s\left(p_{1}\right)=.013, s\left(p_{2}\right)=.015, s\left(p_{3}\right)=$ $.018, s\left(p_{4}\right)=.0 .032$. So we have ranked as follow which give us the overall result of each alternatives. which is in th form of Table- 5 .

Table-5: The ranking results of the suggested techniques Using Algorithm -I.

\begin{tabular}{|c|c|c|c|c|c|}
\hline \multirow[b]{2}{*}{ Proposed Methods } & \multicolumn{4}{|c|}{ score values } & \multirow[b]{2}{*}{ Ranking } \\
\hline & $p_{1}$ & $p_{2}$ & $p_{3}$ & $p_{4}$ & \\
\hline CFHWA & 0.013 & 0.015 & 0.018 & 0.032 & $p_{4}>p_{3}>p_{2}>p_{1}$ \\
\hline CFHWG & 0.012 & 0.014 & 0.024 & 0.034 & $p_{4}>p_{3}>p_{2}>p_{1}$ \\
\hline
\end{tabular}

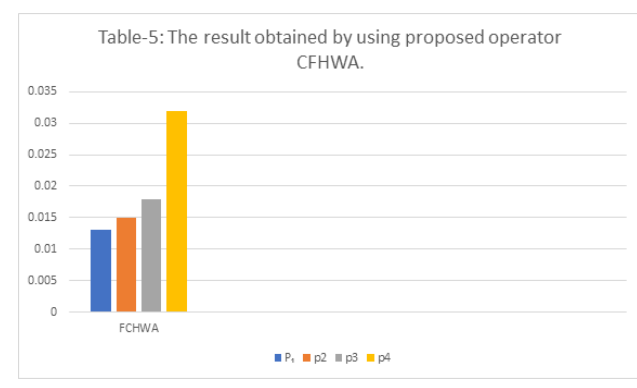




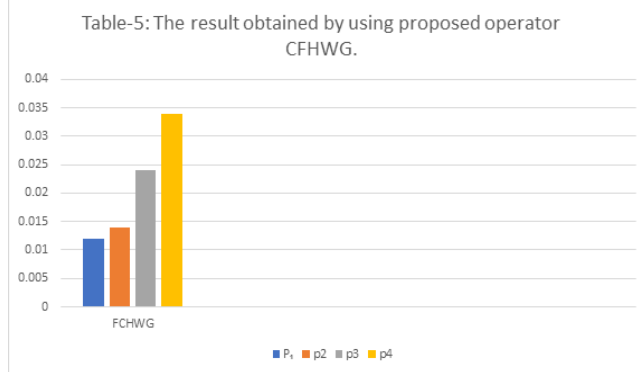

11.19. Illustrative Example Based on Extended GRA Method. Here the steps of proposed method were apply as on the numerical example which is discuss as under. The detail of alternatives, criteria and weight vectors were discussed in detail as above. Also the steps is calculated as under.

Step-1: The first step of the proposed method is that we have to represent the data in the form of matrix shape having alternatives and criteria in the form of FCNs. which is represented inthe form of Table-1, Table-2 and Table-3 as above in the algorithm-I.

Step -2: The second step is about to aggregate all the DM, which will give us a collective DM using the CFHWA/CFHWG which is presented in the form of Table- 4 as in the algorithm-I.

Step-3: The third step of the developed method is to find the PIS and NIS solution by using the formula as above and the result is discuss as in the form of Table- 6 and Table- 7 .

Table-6: The result of Positive Ideal Solution (PIS)

\begin{tabular}{l|c|c|c|c|c}
\hline \hline Alternatives & $c_{1}$ & $c_{2}$ & $c_{3}$ & $c_{4}$ & $c_{5}$ \\
\hline \hline$p_{1}$ & $(0.990,0.983)$ & $(0.988,0.984)$ & $(0.983,0.984)$ & $(0.988,0.984)$ & $(0.982,0.985)$ \\
$p_{2}$ & $(1.000,1.005)$ & $(1.012,1.001)$ & $(1.000,1.004)$ & $(1.004,1.001)$ & $(1.000,1.001)$ \\
$p_{3}$ & $(0.997,0.999)$ & $(0.999,1.005)$ & $(0.997,0.998)$ & $(1.002,0.099)$ & $(1.003,0.997)$ \\
$p_{4}$ & $(1.017,1.005)$ & $(1.002,1.002)$ & $(1.014,1.005)$ & $(0.999,1.001)$ & $(1.007,1.005)$ \\
\hline
\end{tabular}

Table-7: The result of Negative Ideal Solution (NIS)

\begin{tabular}{l|r|r|l|l|c}
\hline \hline Alternatives & $c_{1}$ & \multicolumn{1}{c}{$c_{2}$} & \multicolumn{1}{c}{$c_{3}$} & \multicolumn{1}{c}{$c_{4}$} & \multicolumn{1}{c}{$c_{5}$} \\
\hline \hline$p_{1}$ & $(1,0.992)$ & $(0.998,0.993)$ & $(0.993,0.992)$ & $(0.998,0.993)$ & $(0.992,0.994)$ \\
$p_{2}$ & $(1,1.005)$ & $(1.012,1.001)$ & $(1.001,1)$ & $(1.004,1.001)$ & $(1.000,1.001)$ \\
$p_{3}$ & $(1,1.002)$ & $(1.006,1.003)$ & $(1.003,0.999)$ & $(1.005,1.002)$ & $(1.006,1.004)$ \\
$p_{4}$ & $(1,0.983)$ & $(0.994,0.996)$ & $(0.984,0.996)$ & $(0.982,0.984)$ & $(0.990,0.983)$ \\
\hline
\end{tabular}

Step-4: In this step we have determined the results of Grey Relational Coefficient which is presented in the Table- 8 and Table- 9 .

Table-8: The result of Grey Relational Coefficient.

\begin{tabular}{c|c|c|c|c|c}
\hline \hline PIS $^{+}$ & $c_{1}$ & $c_{2}$ & $c_{3}$ & $c_{4}$ & $c_{5}$ \\
\hline \hline & $(2.004,2.008)$ & $(2.005,2.007)$ & $(2.008,2.007)$ & $(2.005,2.007)$ & $(2.008,2.007)$ \\
& $(1.999,1.996)$ & $(1.993,1.998)$ & $(1.999,1.997)$ & $(1.997,1.998)$ & $(1.999,1.998)$ \\
& $(2.001,1.999)$ & $(2.001,1.999)$ & $(2.001,2.000)$ & $(1.998,1.999)$ & $(1.997,2.008)$ \\
& $(1.990,1.999)$ & $(1.993,1.998)$ & $(1.992,1.999)$ & $(1.999,1.998)$ & $(1.995,1.999)$ \\
\hline
\end{tabular}


Table-9: The result of Grey Relational Coefficient.

\begin{tabular}{c|c|c|c|c|c}
\hline \hline NIS $^{-}$ & $c_{1}$ & $c_{2}$ & $c_{3}$ & $c_{4}$ & $c_{5}$ \\
\hline \hline & $(1.994,1.998)$ & $(1.995,1.997)$ & $(1.998,1.999)$ & $(1.995,1.996)$ & $(1.998,1.997)$ \\
& $(1.994,1.992)$ & $(1.988,1.994)$ & $(1.994,1.992)$ & $(1.992,1.992)$ & $(1.994,1.993)$ \\
& $(2.001,1.999)$ & $(1.994,1.993)$ & $(1.994,1.992)$ & $(1.991,1.992)$ & $(1.991,1.994)$ \\
& $(1.994,2.003)$ & $(1.997,2.002)$ & $(1.996,2.003)$ & $(2.004,2.002)$ & $(2.001,2.003)$ \\
\hline
\end{tabular}

Step-5: In this step we have determined the result of Relative Closeness Coefficient which is listed in the Table-10.

Table-10: The result of Grey Relational Grade.

\begin{tabular}{l|c|c}
\hline \hline Alternatives & $u_{i}^{+}$ & $u_{i}^{-}$ \\
\hline \hline$p_{1}$ & 0.4209 & 0.4189 \\
$p_{2}$ & 0.4812 & 0.4789 \\
$p_{3}$ & 0.4417 & 0.4396 \\
$p_{4}$ & 0.5013 & 0.2993 \\
\hline
\end{tabular}

Step-6: In this step we have to find the ratio of Relative Closeness Coefficient which is denoted by Table-11.

Table-11: The result of Ratio of Grey Relational Coefficient.

\begin{tabular}{l|l}
\hline \hline Alternatives & \multicolumn{1}{|c}{$S_{i}$} \\
\hline \hline$p_{1}$ & 0.4552 \\
\cline { 2 - 2 }$p_{2}$ & 0.4649 \\
\cline { 2 - 2 }$p_{3}$ & 0.5012 \\
\cline { 2 - 2 }$p_{4}$ & 0.5240 \\
\hline
\end{tabular}

The score values of each alternatives is $s\left(p_{1}\right)=0.4552, s\left(p_{2}\right)=0.4649, s\left(p_{3}\right)=$ $0.5012, s\left(p_{4}\right)=0.5240$. So we have ranked as follow which give us the overall result of each alternatives, which is inthe form of Table- 12 .

Table-12: The ranking results of the suggested technique Using Algorithm -II.

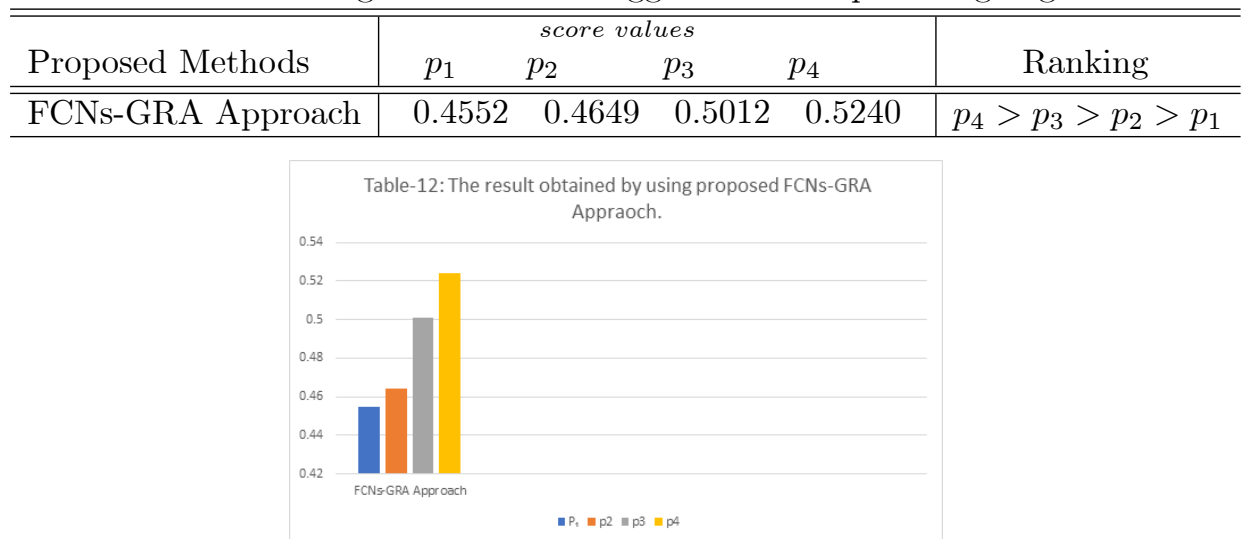

11.20. Verification By TODIM Method. In this subsection we have to verify our proposed approach to check the accuracy and correctness. Here we have taken the data (values) in the form of FCNs and using the Hamacher t-norm and t-conorm, we developed a series of aggregation operator which is CFHWA and CFHWG that is apply to choose the best optimal solution for the given alternatives (selection). So in verification section we have to verify our proposed method with a known 
approach (TODIM approach) and here also to check the results of our proposed method to this known TODIM approach. which give us the result that is the same as using another methods and also which is presented in the Table- 20.

\subsection{Representation of data.}

Step-1: The first step of the proposed method is that we have represent the data in the form of matrix shape having alternatives and criteria. which will be written as.

$$
M=\left[\tau\left(k_{i j}^{l}\right)\right]_{m \times n}
$$

Here using the FCNs also the decision maker is denoted by $D_{i}$. And also $\left(k_{i j}^{l}\right)$ represent the DMs values having alternatives and criteria.

$$
\left.D=\begin{array}{lllll}
c_{1} & c_{2} & c_{3} & \cdots & c_{m} \\
k_{11}^{l} & k_{12}^{l} & k_{13}^{l} & \cdots & k_{1 m}^{l} \\
k_{21}^{l} & k_{22}^{l} & k_{23}^{l} & \cdots & k_{2 m}^{l} \\
\vdots & \vdots & \vdots & \ddots & \vdots \\
k_{n 1}^{l} & k_{n 2}^{l} & k_{n 3}^{l} & \cdots & k_{n m}^{l}
\end{array}\right]
$$

\subsection{Aggregated Decision matrix.}

Step-2: The second step of the proposed methods is to collect DM using proposed aggregation formula which is CFHWA or CFHWG. In this step a $\mathrm{DM}$ is to write as a one (collective matrix) which have an alternative and criteria. And give us an aggregated matrix.

$$
\begin{aligned}
& C F H W G\left(s_{1}, s_{2}, \ldots, s_{n}\right) \\
= & \left\{\otimes_{i=1}^{n} s_{i} w_{i}\right\}^{\varsigma_{i}} \\
= & \left\{\begin{array}{l}
\frac{\lambda \Pi_{i=1}^{n} a_{i}^{w_{i}}}{\Pi_{i=1}^{n}\left(1+(\lambda-1)\left(1-a_{i}\right)\right)^{w_{i}}+(\lambda-1) \Pi_{i=1}^{n} a_{i}^{w_{i}}}, \\
\frac{\Pi_{i=1}^{n}\left(1+(\lambda-1) b_{i}\right)^{w_{i}}-\Pi_{i=1}^{n}\left(1-b_{i}\right)^{w_{i}}}{\Pi_{i=1}^{n}\left(1+(\lambda-1) b_{i}\right)^{w_{i}}+(\lambda-1) \Pi_{i=1}^{n}\left(1-b_{i}\right)^{w_{i}}}
\end{array}\right\}
\end{aligned}
$$

Or

$$
\begin{aligned}
& C F H W A\left(s_{1}, s_{2}, \ldots, s_{n}\right) \\
= & \left\{\oplus_{i=1}^{n} s_{i} w_{i}\right\} \\
= & \left\{\begin{array}{c}
\frac{\Pi_{i=1}^{n}\left(1+(\lambda-1) a_{i}\right)^{w_{i}}-\Pi_{i=1}^{n}\left(1-a_{i}\right)^{w_{i}}}{\Pi_{i=1}^{n}\left(1+(\lambda-1) a_{i}\right)^{w_{i}}+(\lambda-1) \Pi_{i=1}^{n}\left(1-a_{i}\right)^{w_{i}}} \\
\frac{\lambda \Pi_{i=1}^{n} b_{i}^{w_{i}}}{\prod_{i=1}^{n}\left(1+(\lambda-1)\left(1-b_{i}\right)\right)^{w_{i}}+(\lambda-1) \Pi_{i=1}^{n} b_{i}^{w_{i}}}
\end{array}\right\}
\end{aligned}
$$

\subsection{Normalization Of Data.}

Step-3: The third step is to normalize the data in the following way. Also in this step we have to convert the cost criteria into benefit criteria to select the best ranked valued and also to make the data uniform. Another case is that if the data is in the form of benefit type then there is no need of normalization process.

$$
a_{i j}=\left\{\begin{array}{l}
a_{i j}=\left(u_{i j}, v_{i j}\right) \\
a_{i j}^{c}=\left(v_{i j}, u_{i j}\right)
\end{array}\right\}
$$




\subsection{Determination Of Highest weight Values.}

Step-4: In this step we have to find the highest weight $\mathrm{w}_{r}=\max \left\{w_{j} \mid j=\right.$ $1,2, \ldots, n\}$ to the reference criterion. Then we have to find the relative weight $w_{j r}$ of the criterion $\mathrm{c}_{j}$ to the reference criterion as.

$$
w_{j r}=w_{j} / w_{r}
$$

\subsection{Computation Of Dominance Degree Over Other Alternatives.}

Step-5: In this step we have to find the dominance degree of alternative over other alternative $p_{k}$ having the criterion $c_{j}$.

$$
\Phi\left(p_{i}, p_{k}\right)=\left\{\begin{array}{c}
\sqrt{S\left(a_{i j}-a_{j}\right) w_{j r} /\left(\sum_{j=1}^{n} w_{j r}\right)},\left(a_{i j}-a_{j}\right)>0, \\
0, S\left(a_{i j}-a_{j}\right)=0, \\
-\frac{1}{\theta} \sqrt{S\left(a_{i j}-a_{j}\right)\left(\sum_{j=1}^{n} w_{i j}\right) / w_{j r}},\left(a_{i j}-a_{j}\right)<0 .
\end{array}\right\}
$$

Where the attenuation factor of the losses is denoted by $\theta$. If $S\left(a_{i j}-a_{j}\right)>$ $0, \Phi\left(p_{i}, p_{k}\right)$ represent the gain of $p_{i}$ over $p_{k}$ with respect to the criterion $c_{j}$. If $S\left(a_{i j}-a_{j}\right)<0$, Then $\Phi\left(p_{i}, p_{k}\right)$ represent the loss. If $S\left(a_{i j}-a_{j}\right)=0$, Then $\Phi\left(p_{i}, p_{k}\right)$ represent the nill.

\subsection{Determination Of Dominance Degree Of Each Alternatives.}

Step-6: In this step we have to find the dominance degree of each alternatives $p_{i}$ over one of the remaining alternatives $p_{k}$.

$$
\Phi\left(p_{i}, p_{k}\right)=\sum_{j=1}^{n} \Phi_{j}\left(p_{i}, p_{k}\right)
$$

\subsection{Determination Of Overall Dominance Degree.}

Step-7: In this step we have to find the overall dominance degree of each alternatives $p_{i}$.

$$
\zeta\left(p_{i}\right)=\frac{\sum_{k=1}^{m} \Phi\left(p_{i}, p_{k}\right)-\min _{i}\left\{\sum_{k=1}^{m} \Phi\left(p_{i}, p_{k}\right)\right\}}{\max _{i}\left\{\sum_{k=1}^{m} \Phi\left(p_{i}, p_{k}\right)\right\}-\min _{i}\left\{\sum_{k=1}^{m} \Phi\left(p_{i}, p_{k}\right)\right\}}
$$

Where the $0 \leq \zeta\left(p_{i}\right) \leq 1$. The ranking will be find according to the higher values of $\zeta\left(p_{i}\right)$.

\subsection{Ending Of Algorithm.}

Step-8: This is last step of algorithm. 


\subsection{Flow Chart For Algorithm-III}

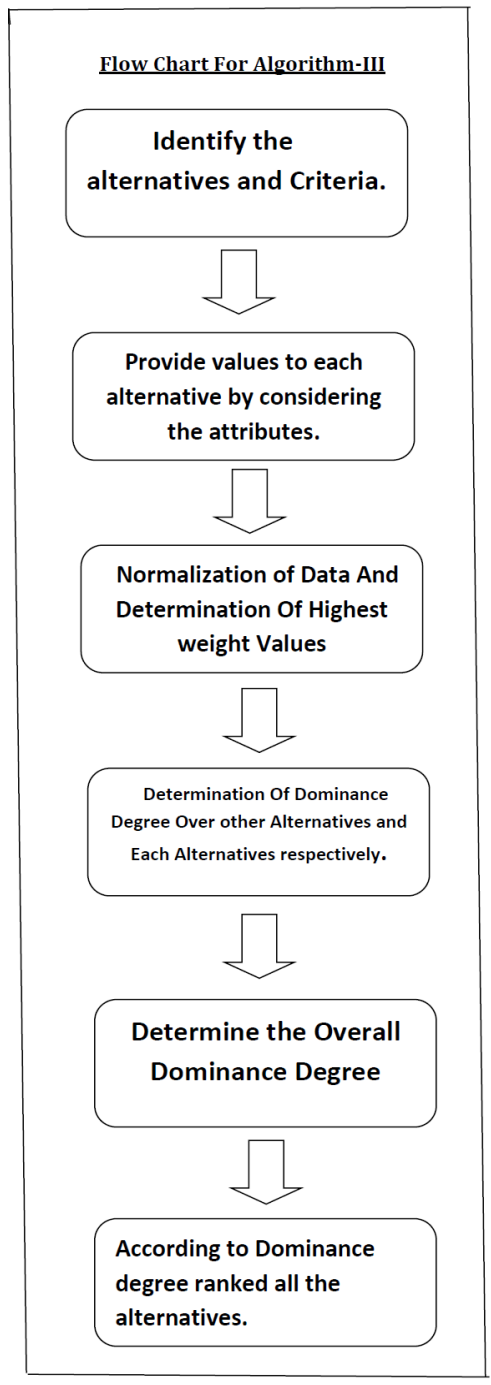

Step-1: The first step of the proposed method is to present the data in the form matrix having alternatives and criteria. The data is presented in the form of Table-1,Table-2 and Table-3 which is given above alogrithm-I.

Step -2: The second step is about to aggregated all the DMs into one collective matrix using the proposed aggregation operators like CFHWA/CFHWG. The result of this step is also given above as we have used these operators to get the required collective DMs, which is represented in the Table- 4 .

Step -3: There is no need of normalization because we have taken the data in the form of benefit and uniform shape. So we have to find the further results (calculation). 
Step -4: In this step of the proposed method we have to find highest weight values. Which is as,

$$
0.875,1,0.916,0.625,0.75
$$

Step -5: In this step we have to find the dominance degree of alternative over other alternative $p_{k}$ having the criterion $c_{j}$. which is listed in the form of Table-13, Table-14, Table-15, Table-16 and Table-17.

Table-13: The result Of Dominance Degree For $c_{1}$.

\begin{tabular}{l|l|l|l|l|l}
\hline \hline Alternatives & \multicolumn{1}{|c|}{$c_{1}$} & \multicolumn{1}{c|}{$c_{2}$} & \multicolumn{1}{c|}{$c_{3}$} & \multicolumn{1}{c|}{$c_{4}$} & \multicolumn{1}{c}{$c_{5}$} \\
\hline \hline$p_{1}$ & $(0,0)$ & $(0.076,0.03)$ & $(0.077,0.28)$ & $(0.12,0.008)$ & $(0.1,0.018)$ \\
$p_{2}$ & $(0.076,0.04)$ & $(0,0)$ & $(0.001,0.26)$ & $(0.176,0.002)$ & $(0.176,0.002)$ \\
$p_{3}$ & $(0.077,0.29)$ & $(0.001,0.02)$ & $(0,0)$ & $(0,0)$ & $(0.177,0.004)$ \\
$p_{4}$ & $(0.1,0.002)$ & $(0.03,0.177)$ & $(0.177,0.288)$ & $(0,0.008)$ & $(0,0)$ \\
\hline
\end{tabular}

Table-14: The result Of Dominance Degree For $\mathrm{c}_{2}$.

\begin{tabular}{l|l|l|l|l|l}
\hline \hline Alternatives & \multicolumn{1}{c|}{$c_{1}$} & \multicolumn{1}{c|}{$c_{2}$} & \multicolumn{1}{c|}{$c_{3}$} & \multicolumn{1}{c}{$c_{4}$} & \multicolumn{1}{c}{$c_{5}$} \\
\hline \hline$p_{1}$ & $(0,0)$ & $(0.06,0.02)$ & $(0.067,0.240)$ & $(0.1,0.03)$ & $(0.17,0.012)$ \\
$p_{2}$ & $(0.06,0.03)$ & $(0,0)$ & $(0.01,0.126)$ & $(0.16,0.02)$ & $(0.13,0.021)$ \\
$p_{3}$ & $(0.07,0.39)$ & $(0.02,0.03)$ & $(0,0)$ & $(0,0)$ & $(0.16,0.042)$ \\
$p_{4}$ & $(0.23,0.01)$ & $(0.04,0.15)$ & $(0.10,0.18)$ & $(0,0.05)$ & $(0,0)$ \\
\hline
\end{tabular}

Table-15: The result Of Dominance Degree For $c_{3}$.

\begin{tabular}{l|l|l|l|l|l}
\hline \hline Alternatives & \multicolumn{1}{c|}{$c_{1}$} & \multicolumn{1}{c|}{$c_{2}$} & \multicolumn{1}{c|}{$c_{3}$} & \multicolumn{1}{c|}{$c_{4}$} & \multicolumn{1}{c}{$c_{5}$} \\
\hline \hline$p_{1}$ & $(0,0)$ & $(0.13,0.10)$ & $(0.37,0.68)$ & $(0.13,0.006)$ & $(0.012,0.018)$ \\
$p_{2}$ & $(0.26,0.14)$ & $(0,0)$ & $(0.013,0.24)$ & $(0.236,0.123)$ & $(0.163,0.021)$ \\
$p_{3}$ & $(0.37,0.29)$ & $(0.26,0.14)$ & $(0,0)$ & $(0,0)$ & $(0.134,0.014)$ \\
$p_{4}$ & $(0.14,0.25)$ & $(0.013,0.27)$ & $(0.227,0.123)$ & $(0.234,0.017)$ & $(0,0)$ \\
\hline
\end{tabular}

Table-16: The result Of Dominance Degree For $\mathrm{c}_{4}$.

\begin{tabular}{l|l|l|l|l|l}
\hline \hline Alternatives & \multicolumn{1}{|c|}{$c_{1}$} & \multicolumn{1}{c|}{$c_{2}$} & \multicolumn{1}{c|}{$c_{3}$} & \multicolumn{1}{c|}{$c_{4}$} & \multicolumn{1}{c}{$c_{5}$} \\
\hline \hline$p_{1}$ & $(0,0)$ & $(0.076,0.03)$ & $(0.077,0.28)$ & $(0.11,0.008)$ & $(0.1,0.017)$ \\
$p_{2}$ & $(0.016,0.02)$ & $(0,0)$ & $(0.001,0.26)$ & $(0.176,0.002)$ & $(0.176,0.002)$ \\
$p_{3}$ & $(0.123,0.096)$ & $(0.001,0.02)$ & $(0,0)$ & $(0,0)$ & $(0.177,0.003)$ \\
$p_{4}$ & $(0.1,0.002)$ & $(0.03,0.14)$ & $(0.177,0.288)$ & $(0,0.008)$ & $(0,0)$ \\
\hline
\end{tabular}

Table-17: The result Of Dominance Degree For $c_{5}$.

\begin{tabular}{l|l|l|l|l|l}
\hline \hline Alternatives & \multicolumn{1}{|c|}{$c_{1}$} & \multicolumn{1}{c|}{$c_{2}$} & \multicolumn{1}{c|}{$c_{3}$} & \multicolumn{1}{c|}{$c_{4}$} & \multicolumn{1}{c}{$c_{5}$} \\
\hline \hline$p_{1}$ & $(0,0)$ & $(0.056,0.03)$ & $(0.097,0.28)$ & $(0.16,0.08)$ & $(0.12,0.016)$ \\
$p_{2}$ & $(0.06,0.04)$ & $(0,0)$ & $(0.01,0.16)$ & $(0.16,0.003)$ & $(0.16,0.028)$ \\
$p_{3}$ & $(0.027,0.29)$ & $(0.01,0.03)$ & $(0,0)$ & $(0,0)$ & $(0.147,0.046)$ \\
$p_{4}$ & $(0.15,0.002)$ & $(0.032,0.16)$ & $(0.15,0.24)$ & $(0.09,0.08)$ & $(0,0)$ \\
\hline
\end{tabular}


Step-6: In this step we have to find the dominance degree of each alternatives $p_{i}$ over one of the remaining alternatives $p_{k}$. which is given in the Table- 18 .

Table-18. The result obtained for dominance degree of each

\begin{tabular}{l|l|l|l|l|l}
\hline \hline Alternatives & \multicolumn{1}{|c|}{$c_{1}$} & \multicolumn{1}{c|}{$c_{2}$} & \multicolumn{1}{c|}{$c_{3}$} & \multicolumn{1}{c|}{$c_{4}$} & \multicolumn{1}{c}{$c_{5}$} \\
\hline \hline$p_{1}$ & $(0,0)$ & $(0.398,0.021)$ & $(0.688,1.48)$ & $(0.62,0.132)$ & $(0.502,0.081)$ \\
$p_{2}$ & $(0.472,0.27)$ & $(0,0)$ & $(0.035,1.04)$ & $(0.908,0.15)$ & $(0.805,0.074)$ \\
$p_{3}$ & $(0.667,1.356)$ & $(0.292,0.24)$ & $(0,0)$ & $(0,0)$ & $(0.795,0.136)$ \\
$p_{4}$ & $(0.72,0.266)$ & $(0.145,0.89)$ & $(0.831,1.11)$ & $(0.324,0.163)$ & $(0,0)$ \\
\hline
\end{tabular}

Step-7: In this step we have to find the overall dominance degree of each alternatives which is presented in the Table-19. And also on the basis of this step we have to ranked all the alternatives.

Table -19. The result using Extended TODIM technique.

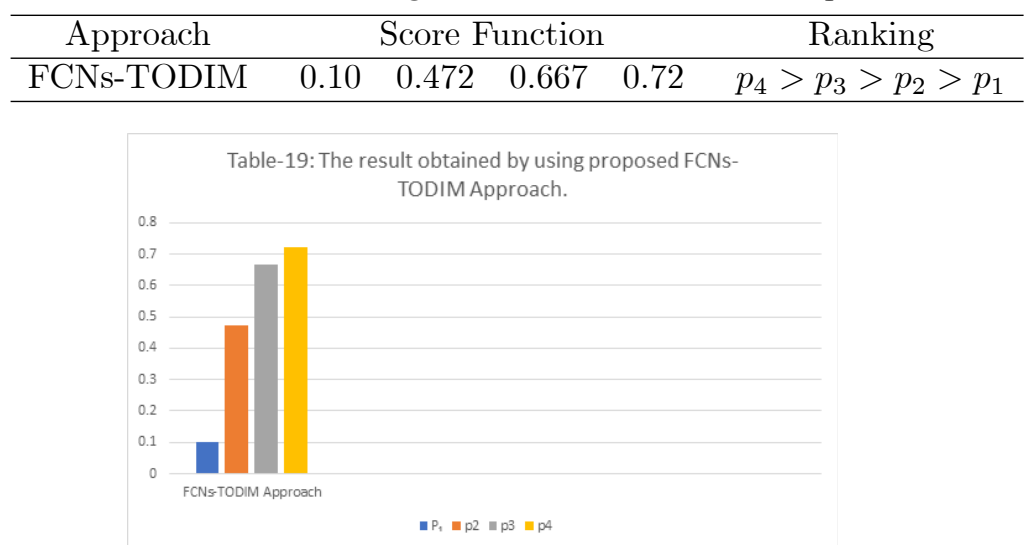

Step-7: In this step we have to find the overall dominance degree of each alternatives $p_{i}$. And also the ranking is find out through proper proposed methods. which is as follow.

$$
p_{4}>p_{3}>p_{2}>p_{1}
$$

11.30. Comparative Study. In this subsection we have to compare our proposed method with other existing methods. In the proposed method we have taken the values in the form of FCNs and also using the concept of Hamacher t-norm and t-conorm we have proposed some aggregation operators like CFHWA/CFHWG. Further more we have to select the best alternatives (option) from given alternatives (options) we can used the score and accuracy function. Now to check the validity and accuracy of the proposed method we have to compare our results with other results. Due to this series of aggregation operators we have solve a numerical example which give us the best results. Also we have explained the modified GRA methods.

But in this paper we have to compare our proposed methods with other existing methods in two way (form) of comparison which is as to compare with aggregation operators like IFWA, IFWG, IFDWA and IFDWG. And the second way (form) of comparison is to compare our results with other results is the fuzzy techniques (approaches) like IF-TOPSIS methods etc. [44, 45, 46, 47]. So a comparison with 
these types of data we can see that our proposed method is best and accurate. The results and comparison analysis details as under.

11.31. Comparison with IFWA. In this subsection we can compare our new proposed work with the existing methods. In this existing methods the basic idea were IFS and their basic aggregation operators like IFWA, [44] IFOWA [44] and IFHWA [44]. But here in this paper we can take the data in the form of FCNs and basic operation of Hamacher t-norm and t-conorm. Further we can also extend and proposed the new aggregation operators like CFHWA, CFHOWA and CFHHWA to aggregate the MAGDM issues and new score and accuracy function were also defined which can help in the final ranking of the object which is in the best position. As in existing methods Z.S et al. [44] take the values in the form IFSs which contain membership degree and non membership degree and also fulfilled the condition that sum of its lies between zero and one. But here in this paper we have taken the values as FCNs and by comparing this proposed work with the existing we can see that our proposed work is more accurate and more applicable than existing methods. The results is shown as follows.

\begin{tabular}{|c|c|c|}
\hline Approaches & Score values & Ranking \\
\hline IFWA [44] & & $p_{4}>p_{3}>p_{2}>p_{1}$ \\
\hline CFHWA & $\begin{array}{llll}0.013 & 0.015 & 0.018 & 0.032\end{array}$ & $p_{4}>p_{3}>p_{2}>p_{1}$ \\
\hline
\end{tabular}

11.32. Comparison with IFWG. We can compare our new proposed work with the existing methods in this subsection. In this existing methods the basic idea were IFS and their basic aggregation operators like IFWG, [45] IFOWG [45] and IFHWG [45]. But here in this paper we can take the data in the form of FCNs and basic operation of Hamacher t-norm and t-conorm. Further we an also extend and proposed the new aggregation operators like CFHWG, CFHOWG and CFHWG to aggregate the MAGDM issues and a score and accuracy function were also defined which can help in the final ranking of the object which is in the best position. As in existing methods Z.S et al. [45] take the values in the form IFSs which contain membership degree and non membership degree and also fulfilled the condition that sum of its lies between zero and one. But here in this paper we have taken the values as FCNs and by comparing this proposed work with the existing we can see that our proposed work is more accurate and more applicable than existing methods. The results is shown as follows.

\begin{tabular}{|c|c|c|}
\hline Approaches & Score values & Ranking \\
\hline IFWG [45] & & $p_{4}>p_{3}>p_{2}>p_{1}$ \\
\hline CFHWG & $\begin{array}{llll}0.012 & 0.014 & 0.024 & 0.034\end{array}$ & $p_{4}>p_{3}>p_{2}>p_{1}$ \\
\hline
\end{tabular}

11.33. Comparison with IFDWA/IFWDG. We can compare our new proposed work with the existing methods in this subsection . In this existing methods the basic idea were IFS and their basic aggregation operators like IFDWA, [46] IFDOWA [46] and IFDHWA [46] IFDWG, [46] IFDOWG [46] and IFDHWG [46] using the basic concept of Dombi t-norm and t-conorm. but here in this paper we can take the data in the form of FCNs and basic operation of Hamacher t-norm and t-conorm. Further we an also extend and proposed the new aggregation operators like CFHWA, CFHOWA and CFHHWA, CFHWG, CFHOWG and CFHHG to aggregate the MAGDM issues and a score and accuracy function were also defined which can help in the final ranking of the object which is in the best position. 
As in existing methods M. R. Seikh et al. [46] take the values in the form IFSs which contain membership degree and non membership degree and also fulfilled the condition that sum of its lies between zero and one. But here in this paper we have taken the values as FCNs and by comparing this proposed work with the existing we can see that our proposed work is more accurate and more applicable than existing methods. The results is shown as follows.

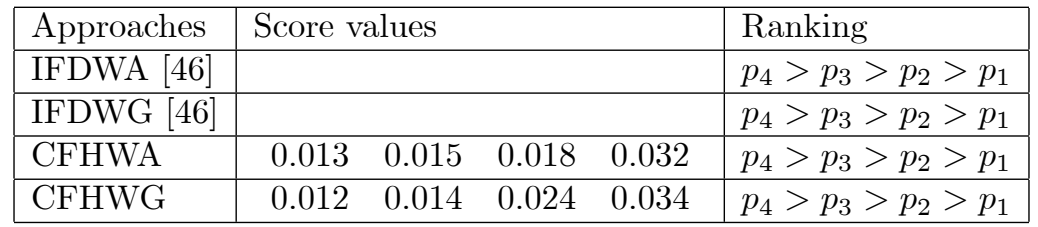

11.34. Comparison with IF-TOPSIS Method. We can compare our new proposed work with the existing methods in this subsection. In this existing methods a generalized IFRSs and its basic aggregation operators will discussed as well as the rough set and its upper and lower approximation is also presented. but here in this paper we can take the data in the form of FCNs and basic operation of Hamacher t-norm and t-conorm. Further we an also extend and proposed the new aggregation operators like CFHWA, CFHOWA and CFHHWA, CFHWG, CFHOWG and CFHHG to aggregate the MAGDM issues and a score and accuracy function were also defined which can help in the final ranking of the object which is in the best position. As in existing methods Z. Zhang [47] proposed IFSs, rough set and also the lower and upper approximation space using these basic operational laws of FCNs were presented. But here in this paper we have taken the values as FCNs and by comparing this proposed work with the existing we can see that our proposed work is more accurate and more applicable than existing methods. The results is shown as follows

\begin{tabular}{|l|lrrr|l|}
\hline Approaches & \multicolumn{3}{|l|}{ Score values } & Ranking \\
\hline IF-TOPSIS [47] & $\times$ & $\times$ & $\times$ & $\times$ & $p_{4}>p_{3}>p_{2}>p_{1}$ \\
\hline FCNs-TODIM Approach & 0.10 & 0.472 & 0.667 & 0.72 & $p_{4}>p_{3}>p_{2}>p_{1}$ \\
\hline FCNs-GRA Approach & 0.455 & 0.46 & 0.501 & 0.52 & $p_{4}>p_{3}>p_{2}>p_{1}$ \\
\hline
\end{tabular}

Table-20. The comparison analysis with other existing methods.

\begin{tabular}{|c|c|c|}
\hline \multirow[b]{2}{*}{ Suggested Techniques } & \multicolumn{2}{|l|}{ score values } \\
\hline & $\begin{array}{llll}p_{2} & p_{4} & p_{1} & p_{3} \\
\end{array}$ & Ranking \\
\hline IFWA & Invisible & $p_{4}>p_{3}>p_{2}>p_{1}$ \\
\hline IFWG & Invisible & $p_{4}>p_{3}>p_{2}>p_{1}$ \\
\hline IFDWG & Invisible & $p_{4}>p_{3}>p_{2}>p_{1}$ \\
\hline IFDWA & Invisible & $p_{4}>p_{3}>p_{2}>p_{1}$ \\
\hline IF-TOPSI & Invisible & $p_{4}>p_{3}>p_{2}>p_{1}$ \\
\hline FCNs-TODIM Approach & $\begin{array}{llll}0.10 & 0.47 & 0.66 & 0.72 \\
\end{array}$ & $p_{4}>p_{3}>p_{2}>p_{1}$ \\
\hline FCNs-GRA Approach & $\begin{array}{llll}0.45 & 0.46 & 0.50 & 0.52 \\
\end{array}$ & $p_{4}>p_{3}>p_{2}>p_{1}$ \\
\hline CFHWA Proposed & $\begin{array}{llll}0.013 & 0.015 & 0.018 & 0.032 \\
\end{array}$ & $p_{4}>p_{3}>p_{2}>p_{1}$ \\
\hline CFHWG Proposed & $\begin{array}{llll}0.012 & 0.014 & 0.024 & 0.034\end{array}$ & $p_{4}>p_{3}>p_{2}>p_{1}$ \\
\hline
\end{tabular}

11.35. Result and discussion. The result and outcome of our proposed work is that we have taken the data in the form of FCNs and also using the Hamacher tnorm and t-conorm basic operational laws we have developed a series of aggregation 
operators like CFHWA and CFHWG operators. Furthermore we have applied these aggregation operators to a MCGDM issues to select the best option from a given data. Also here in this paper we have developed and explained a stepwise algorithm in which some steps is defined and also calculated for the numerical case like a practical example of electricity crises in Pakistan. Further the discussion of this paper is that we have also compare our proposed work to other existing methods to check the accuracy and accurateness of the proposed methods. And in lastly we have verified our proposed work by the other existing approach to study the correctness of the new proposed approach.

\section{Conclusion And Future Work}

Normally there are many types of decision making problems but we have focused only two types which are multi criteria decision making problems(MCDM) and other is multi criteria group decision making problems(MCGDM) during any decision problems. But here in this paper we have taken the MCGDM problems and get the best results on the basis of our proposed work which is also define on the basis of Hamacher t-norm and t-conorm and as a result we achieve a series of aggregation operators like CFHWA and CFHWG. Also more in this paper we have to defined and explained the modified GRA and TODIM method using the FCNs and the detail description of our proposed method is also explained in the section of paper. Finally a comparison with other methods is done to check the accuracy of our new work. And a numerical example is solved and verify by a known (exists) method, which give us the same and best result which is achieve by our proposed work. Also in this paper using the FCNs the main benefit is that its provide a credible and accurate degree to the DM problems. In future work this study may be applied to the many practical applications like Dombi t-norm and t-conorm and Yager t-norm and t-conorm to select the best possible outcome using the FCNs.

\section{REFERENCES}

[1] L. A. Zadeh, "Fuzzy sets," Inf Control., Vol. 8, no. 3, pp. 338-353, 1965.

[2] Ye, J., Song, J., Du, S. and Yong, R., 2021. Weighted aggregation operators of fuzzy credibility numbers and their decision-making approach for slope design schemes. Computational and Applied Mathematics, 40(4), pp.1-14.

[3] J.L. Deng, Introduction to Grey System, The Journal of Grey System (UK) 1 (1) (1989) $1-24$.

[4] J.L. Deng, Grey System Theory, Press of Huazhong University of Science and Technology, Wuhan, 2002.

[5] S. F. Liu, T. B. Guo, Y. G. Dang, Grey System Theory and its Application (Second Edition). Beijing: Science Press, 1999, 26-29.

[6] G. W. Wei, Grey relational analysis method for 2-tuple linguistic multiple attribute group decision making with incomplete weight information, Expert Systems with Applications, 2010. (in press)

[7] G. W. Wei, X.F. Zhao, H.J. Wang and R. Lin, GRA model for selecting an ERP system in trapezoidal intutionistic fuzzy setting, Information: An International Journal 13(4)(2010) $1143-1148$

[8] G. W. Wei, R. Lin, X.F Zhao and H.J. Wang, TOPSIS-based linear-programming methodology for multiple attribute decision making with incomplete weight information in intuitionistic fuzzy setting, Information: An International Journal 13(5)(2010).

[9] D.L. Olson, D.S.Wu, Simulation of fuzzy multi-attribute models for grey relationships, European Journal of Operational Research 175 (11) (2006) 111-120. 
[10] D. S. Wu, Supplier selection in a fuzzy group decision making setting: A method using grey related analysis and Dempster-Shafer theory, Expert Systems with Applications 36(2009) 8892-8899.

[11] C. J. Rao, X.P. Xiao, Novel Combinatorial Algorithm for the Problems of Fuzzy Grey Multiattribute Group Decision Making, Journal of Systems Engineering and Electronics 18(4) (2007) 774-780.

[12] C. J. Rao, Y. Zhao, Multi-attribute Decision Making Model Based on Optimal Membership and Relative Entropy, Journal of Systems Engineering and Electronics 20(3) (2009) 537-542.

[13] C. J. Rao, Group Decision Making Model Based on Grey Relational Analysis. The Journal of Grey System 21(1) (2009) 15-24.

[14] C. J. Rao, Y. Zhao, Multi-attribute Auction Method Based on Grey Relational Degree of Hybrid Sequences, The Journal of Grey System 21(2) (2009) 175-184.

[15] G. W. Wei, Uncertain linguistic hybrid geometric mean operator and its Application to group decision making under uncertain linguistic environment, International Journal of Uncertainty, Fuzziness and Knowledge-Based Systems 17(2) (2009) 251-267.

[16] G. W. Wei, A method for multiple attribute group decision making based on the ET-WG and ET-OWG operators with 2-tuple linguistic information, Expert Systems with Applications $37(12)(2010) 7895-7900$.

[17] X.B. Li, D. Ruan, J. Liu and Y. Xu, A linguistic-valued weighted aggregation operator to multiple attribute group decision making with quantitative and qualitative information, International Journal of Computational Intelligence Systems 1(3) (2008) 274-284.

[18] G. W. Wei, R. Lin, X.F. Zhao, H.J. Wang, Models for multiple attribute group decision making with 2-tuple linguistic assessment information, International Journal of Computational Intelligence Systems 3(3) (2010) 315-324.

[19] X.B. Li, D. Ruan, J. Liu, Y. Xu, A linguistic-valued weighted aggregation operator to multiple attribute group decision making with quantitative and qualitative information, International Journal of Computational Intelligence Systems 1(3) (2008) 274-284.

[20] G.Q. Zhang, C.G. Shi, J. Lu, An extended KTH-Best approach for referential-uncooperative bi level multi-follower decision making, International Journal of Computational Intelligence Systems 1(3) (2008) $205-214$.

[21] C. Kahraman, A. C. Tolga, An alternative ranking approach and Its usage in multi-criteria decision-making, International Journal of Computational Intelligence Systems 2(3) (2009).

[22] G. W. Wei, Extension of TOPSIS method for 2-tuple linguistic multiple attribute group decision making with incomplete weight information, Knowledge and Information Systems 25 (2010) 623-634.

[23] J. Ma, D. Ruan, Y. Xu and G. Zhang, A fuzzy-set approach to treat determinacy and consistency of linguistic terms in multi-criteria decision making, International Journal of Approximate Reasoning, 44(2)(2007)165-181.

[24] L. Martinez, J. Liu, D. Ruan, J.B Yang, Dealing with heterogeneous information in engineering evaluation processes, Information Sciences 177(7) (2007) 1533-1542.

[25] L. Martinez, D. Ruan, F. Herrera, E. Herrera-Viedma, P.P. Wang, Linguistic decision making: Tools and applications, Information Sciences 179(14) (2009) 2297-2298.

[26] L. F. A. M. Gomes and M. M. P. P. Lima, "TODIM: basics and application to multicriteria ranking of projects with environmental impacts," Foundations of Computing and Decision Sciences, vol. 16, no. 4, pp. 113-127, 1992.

[27] L. F. A. M. Gomes and M. M. P. P. Lima, "From modeling individual preferences to multicriteria ranking of discrete alternatives: a look at prospect theory and the additive difference model," Foundations of Computing and Decision Sciences, vol.17, no. 3, pp. 171-184, 1992.

[28] D. Kahneman and A. Tversky, "Prospect theory: an analysis of decision under risk," Econometrica, vol. 47 , no. 2, p. 263, 1979.

[29] L. F. A. M. Gomes and L. A. D. Rangel, "An application of the TODIM method to the multicriteria rental evaluation of residential properties," European Journal of Operational Research, vol. 193, no. 1, pp 204-211, 2009.

[30] R. A. Krohling and T. T. M. De Souza, "Combining prospect theory and fuzzy numbers to multi-criteria decision making," Expert Systems with Applications, vol. 39, no. 13, pp. 11487-11493, 2012

[31] Z.-P. Fan, X. Zhang, F.-D. Chen, and Y. Liu, "Extended TODIM method for hybrid multiple attribute decision making problems," Knowledge-Based Systems, vol. 42, pp. 40-48, 2013. 
[32] X.-W. Qi, C.-Y. Liang, E.-Q. Zhang, and Y. Ding, "Approach to interval-valued intuitionistic fuzzy multiple attributes group decision making based on maximum entropy," System Engineering Theory and Practice, vol. 31, no. 10, pp. 1940-1948, 2011.

[33] Zhou F, Chen TY. A novel distance measure for Pythagorean fuzzy sets and its applications to the technique for order preference by similarity to ideal solutions. Comput Intell Syst. 2019;12(2): 955-969.

[34] Mohagheghi V, Mousavi SM, Vahadani B. Enhancing decision-making flexibility by introducing a new last aggregation evaluating approach based on multi-criterion group decision making and Pythagorean fuzzy sets. Appl Soft Comput. 2017;61:527-535.

[35] Beliakov G, Pradera A, Calvo T. Aggregation Functions: A Guide For Practitioners. Springer, Heidelberg; 2007.

[36] Augustine P. Pythagorean fuzzy set and its application in career placement based on academic performance using max-min composition. Complex Intell Syst. 2019;5:165-175.

[37] Tan CQ, Yi WT, Chen XH. Hesitant fuzzy Hamacher aggregation operators for multicriteria decision making. Appl Soft Comput. 2015;26:325-349.

[38] Senapati T, Yager RR. Some new operations over Fermatean fuzzy numbers and application of Fermatean fuzzy WPM in multiple criteria decision making. Informatica. 2019;30(2):391-412.

[39] Merigó JM, Gil-Lafuente AM (2011) Fuzzy induced generalized aggre gation operators and its application in multi-person decision making. Expert Syst Appl 38(8):9761-9772.

[40] Song Q, Kandel A, Schneider M (2003) Parameterized fuzzy operators in fuzzy decision making. Int J Intell Syst 18(9):971-987.

[41] G. Deschrijver, C. Cornelis and E.E. Kerre, On the representation of intuitionistic fuzzy t-norms and t-conorms, IEEE Transactions on Fuzzy Systems 12 (2004), 45-61.

[42] S. Roychowdhury and B.H. Wang, On generalized Hamacher families of triangular operators, International Journal of Approximate Reasoning 19 (1998), 419-439.

[43] G. Deschrijver and E.E. Kerre, A generalization of operators on intuitionistic fuzzy sets using triangular norms and t-conorms, Notes on Intuitionistics Fuzzy Sets 8 (2002), 19-27.

[44] Z. S. Xu, "Intuitionistic fuzzy aggregation operators," IEEE Trans Fuzzy Syst., vol. 15: pp. 1179-1187, 2007.

[45] Z. S. Xu, and R. R. Yager, "Some geometric aggregation operators based on intuitionistic fuzzy sets," Int J Gen Syst., vol. 35,pp. 417-433, 2006.

[46] M. R. Seikh, and U. Mandal, "Intuitionistic fuzzy Dombi aggregation operators and their application to multiple attribute decision-making," Granular Comput., pp. 1-16, 2019.

[47] Joshi, D. and Kumar, S., 2014. Intuitionistic fuzzy entropy and distance measure based TOPSIS method for multi-criteria decision making. Egyptian informatics journal, 15(2), pp.97-104.

Abdul Wali Khan University Mardan.

E-mail address: saleemabdullah@awkum.edu.pk

Abdul Wali Khan University Mardan.

E-mail address: yahya@akwum.edu.pk 